\title{
Properties of singularities in (phantom) dark energy universe
}

\author{
Shin'ichi Nojiri,, , 田 Sergei D. Odintsov, ${ }^{2, \text { I }}$ and Shinji Tsujikawa, \\ ${ }^{1}$ Department of Applied Physics, National Defence Academy, Hashirimizu Yokosuka 239-8686, Japan \\ ${ }^{2}$ Instituciò Catalana de Recerca $i$ Estudis Avanats (ICREA) and Institut d'Estudis Espacials de Catalunya (IEEC), \\ Edifici Nexus, Gran Capità 2-4, 08034 Barcelona, Spain \\ ${ }^{3}$ Department of Physics, Gunma National College of Technology, Gunma 371-8530, Japan
}

(Dated: February 1, 2008)

\begin{abstract}
The properties of future singularities are investigated in the universe dominated by dark energy including the phantom-type fluid. We classify the finite-time singularities into four classes and explicitly present the models which give rise to these singularities by assuming the form of the equation of state of dark energy. We show the existence of a stable fixed point with an equation of state $w<-1$ and numerically confirm that this is actually a late-time attractor in the phantomdominated universe. We also construct a phantom dark energy scenario coupled to dark matter that reproduces singular behaviors of the Big Rip type for the energy density and the curvature of the universe. The effect of quantum corrections coming from conformal anomaly can be important when the curvature grows large, which typically moderates the finite-time singularities.
\end{abstract}

PACS numbers: $98.70 . \mathrm{Vc}$

\section{INTRODUCTION}

The increasing evidence from the observational data indicates that (linear) equation of state (EOS) parameter $w$ lies in a narrow strip around $w=-1$ quite likely being below of this value [1]. The region where the EOS parameter $w$ is less than -1 is typically referred as a phantom dark energy universe. This is caused by the fact that when the phantom EOS is constructed in terms of a scalar field the corresponding kinetic term is chosen to have a wrong sign (negative kinetic energy). Of course, this is not the only possibility: the phantom-like value for $w$ may appear from Brans-Dicke (BD) scalartensor gravity, from non-standard (negative) potentials, from the non-minimal coupling of scalar Lagrangian with gravity or even usual matter may appear in phantomlike form. Recent works [2, 3, 44 have been devoted to the study of phantom cosmologies produced by different models.

The existence of the region with $w<-1$ (if such a phase in the universe evolution indeed occurs) opens up a number of fundamental questions. For instance, the entropy of such universe is negative (or the characteristic temperatures should be negative). The dominant energy condition (DEC) for phantom matter is violated, as a rule. The phantom dominated universe ends up with a finite-time future singularity called Big Rip or Cosmic Doomsday (see Refs. [5, 6]). This last property attracted much attention and brought the number of speculations up to the explicit calculation of the rest life-time of our universe! In its turn, such a study motivated the mathematical investigation of singularities when the DEC or

\footnotetext{
*Electronic address: snojiri@yukawa.kyoto-u.ac.jp

${ }^{\dagger}$ Electronic address: odintsov@aliga.ieec.uab.es

${ }^{\ddagger}$ Electronic address: shinji@nat.gunma-ct.ac.jp
}

the strong energy condition (SEC) is violated 7] (see also Ref. [8]). However, the Big Rip singularity is characterized by the growth of the energy and curvature invariants with a divergent scale factor at Big Rip time. The energy scale may grow up to Planck one, giving rise to the second Quantum Gravity era. Eventually, quantum effects become important near the singularity where they may moderate or even prevent the singularity [6, [9] .

In such circumstances, it is fundamentally important to understand the properties of singularities in phantom dark energy universe, to classify them and to search for realistic ways to avoid the singularities. The present article is devoted to the investigation of the above circle of problems. We study phenomenological models in which the pressure density $p$ of dark energy is given in terms of the function of the energy density $\rho$, i.e., $p=-\rho-f(\rho)$. When the function $f(\rho)$ is zero, the EOS parameter $w$ is equal to -1 (cosmological constant EOS). We classify the types of future singularities and show explicit examples of phantom dark energy which realize all mentioned types of singularities. The evolution of such dark energy universe at late times (near to singularity) is investigated both analytically and numerically.

The paper is organized as follows. The general system of dark energy coupled with dark matter is considered in Sec. II. The corresponding background equations are written in autonomous form which permits to analyze the fixed points and attractor solutions. We also study the stability of fixed points against perturbations and show the existence of a stable critical point with $w<-1$. Sec. III is devoted to the classification of finite-time future singularities (four different types) and their explicit realization in terms of the universe with the equation of state: $p=-\rho-f(\rho)$. We present the existence of singularities of much less explicit type than the standard Big Rip where the scale factor is finite at Rip time (including the type given in Ref. 7] ). We construct examples of phantom dark energy universes in which all four types of 
singularities appear. The violation of strong and dominant energy conditions is investigated as well. We also present one example of the model which admits the transition from $w<-1$ to $w>-1$. In Sec. IV the relation between the EOS function $f(\rho)$ and the appearance of singularities is studied. Sec. $\mathrm{V}$ is devoted to the numerical study of attractor solutions in dark energy models with non-relativistic dark matter. The phase plane analysis confirms the structure of singularities and the stability around the fixed points.

In Sec. VI we present coupled phantom/fluid dark matter models for several specific coupling functions. These models generalize some of the examples of the previous sections. We explicitly show multiple scalar field models that exhibit the similar singular structure as in the coupled phantom scenario. In Sec. VII the role of quantum effects is investigated for the types of singularities classified in Sec. III. In accordance with previous attempts in this direction [6, 9, 10] it is demonstrated that quantum effects can moderate the finite-time singularities. Summary and some outlook are given in the final section.

\section{AUTONOMOUS SYSTEM}

Let us consider a system with two fluids: (i) dark energy with an equation of state: $p=p(\rho)$, and (ii) a barotropic perfect fluid with an equation of state: $p=w_{m} \rho$. We wish to study the case of a nonrelativistic dark matter $\left(w_{m}=0\right)$, but we keep our discussion in general for the moment. In a spatially flat FriedmannLemaitre-Robertson-Walker (FLRW) metric with a scale factor $a$, the background equations are given by

$$
\begin{aligned}
& \dot{\rho}+3 H(\rho+p)=-Q, \\
& \dot{\rho}_{m}+3 H\left(\rho_{m}+p_{m}\right)=Q, \\
& \dot{H}=-\frac{\kappa^{2}}{2}\left(\rho+p+\rho_{m}+p_{m}\right),
\end{aligned}
$$

where $H \equiv \dot{a} / a$ is the Hubble rate, and $\kappa^{2} \equiv 8 \pi G$ with Newton's gravitational constant $G$. Here a dot denotes the derivative with respect to cosmic time $t$. We accounted for the interaction term $Q$ between dark energy and the barotropic fluid. We also have the constraint equation for the Hubble rate:

$$
H^{2}=\frac{\kappa^{2}}{3}\left(\rho+\rho_{m}\right) .
$$

In analogy with scalar field dark energy models, we introduce the following "kinematic" and "potential" terms:

$$
\rho_{K} \equiv(\rho+p) / 2, \quad \rho_{V} \equiv(\rho-p) / 2 .
$$

For scalar fields one has $\rho=\frac{1}{2} \epsilon \phi^{2}+V(\phi)$ and $p=$ $\frac{1}{2} \epsilon \phi^{2}-V(\phi)$ (a normal field corresponds to $\epsilon=1$ and a phantom to $\epsilon=-1)$. We shall introduce the following dimensionless quantities:

$$
x \equiv \frac{\kappa^{2} \rho_{K}}{3 H^{2}}, \quad y \equiv \frac{\kappa^{2} \rho_{V}}{3 H^{2}} .
$$

Then the above background equations can be written in an autonomous form as an extension of Ref. 11]:

$$
\begin{aligned}
\frac{\mathrm{d} x}{\mathrm{~d} N}= & -\left[1+p^{\prime}(\rho)\right]\left[3+\frac{Q}{2 H \rho_{K}}\right] x \\
& +3 x\left[2 x+\left(1+w_{m}\right)(1-x-y)\right], \\
\frac{\mathrm{d} y}{\mathrm{~d} N}= & -\left[1-p^{\prime}(\rho)\right]\left[3 x+\frac{Q}{2 H \rho_{V}} y\right] \\
& +3 y\left[2 x+\left(1+w_{m}\right)(1-x-y)\right], \\
\frac{1}{H} \frac{\mathrm{d} H}{\mathrm{~d} N}= & -\left[3 x+\frac{3}{2}\left(1+w_{m}\right)(1-x-y)\right],
\end{aligned}
$$

together with the constraint

$$
\Omega_{m} \equiv \frac{\kappa^{2} \rho_{m}}{3 H^{2}}=1-x-y,
$$

where $N \equiv \ln a$ and $p^{\prime}(\rho)$ is a function of $\rho$ defined as $p^{\prime}(\rho) \equiv \mathrm{d} p / \mathrm{d} \rho$. Since $\kappa^{2} \rho_{m} /\left(3 H^{2}\right) \geq 0$, the parameter range of $x$ and $y$ is restricted to be

$$
x+y \leq 1
$$

When $w_{m}=0$ and $Q=0$, these are simplified as

$$
\begin{aligned}
& \frac{\mathrm{d} x}{\mathrm{~d} N}=3 x\left[x-y-p^{\prime}(\rho)\right], \\
& \frac{\mathrm{d} y}{\mathrm{~d} N}=-3\left[1-p^{\prime}(\rho)\right] x+3 y[1+x-y], \\
& \frac{1}{H} \frac{\mathrm{d} H}{\mathrm{~d} N}=-\frac{3}{2}(1+x-y) .
\end{aligned}
$$

Let us consider a situation in which $p^{\prime}(\rho)$ asymptotically approaches a constant, i.e., $p^{\prime}(\rho) \rightarrow w$. In this case the equation of state for dark energy is

$$
w \equiv \frac{p}{\rho}=\frac{x-y}{x+y} .
$$

In the phase plane in terms of $x$ and $y$, the trajectory corresponding to the constant $w$ is a straight line, i.e.,

$$
y=\frac{1-w}{1+w} x .
$$

Setting $\mathrm{d} x / \mathrm{d} N=0$ and $\mathrm{d} y / \mathrm{d} N=0$ in Eqs. (12) and (13), one obtains the following fixed points: (i) $(x, y)=$ $(0,0)$, (ii) $(x, y)=[(1+w) / 2,(1-w) / 2]$ and (iii) $(x, y)=$ $(0,1)$. The fixed point (i) corresponds to the barotropic fluid dominant solution $\left(\Omega_{m} \rightarrow 1\right)$. The point (ii) is the dark energy dominant solution, that is, $\Omega_{\mathrm{DE}} \equiv x+y \rightarrow 1$. In the case (ii) we have

$$
\frac{1}{H} \frac{\mathrm{d} H}{\mathrm{~d} N}=-\frac{3}{2}(1+w),
$$

which means that the Hubble rate increases for $w<-1$. The class (iii) corresponds to the potential-dominant solution with $\Omega_{\mathrm{DE}} \equiv x+y \rightarrow 1$.

One can investigate the stability of the system by considering small perturbations $\delta x$ and $\delta y$ around the fixed 
point $\left(x_{0}, y_{0}\right)$, i.e., $x=x_{0}+\delta x$ and $y=y_{0}+\delta y$. By Eqs. (12) and (13), we obtain the linearized equations

$$
\begin{aligned}
\frac{\mathrm{d}}{\mathrm{d} N} \delta x & =3\left(2 x_{0}-y_{0}-w\right) \delta x-3 x_{0} \delta y, \\
\frac{\mathrm{d}}{\mathrm{d} N} \delta y & =3\left(-1+y_{0}+w\right) \delta x+3\left(1+x_{0}-2 y_{0}\right) \delta y,
\end{aligned}
$$

This can be written by using a matrix $\mathcal{M}$ :

$$
\frac{\mathrm{d}}{\mathrm{d} N}\left(\begin{array}{l}
\delta x \\
\delta y
\end{array}\right)=\mathcal{M}\left(\begin{array}{l}
\delta x \\
\delta y
\end{array}\right)
$$

One can study the stability of critical points against perturbations by evaluating the eigenvalues of the matrix $\mathcal{M}$. For the class (i) one has $\lambda=-3 w$ and $\lambda=3$, which means that the solution is an unstable node for $w<0$ and a saddle point for $w>0$. For the class (ii) we obtain $\lambda=3 w$ and $\lambda=3(w+1)$. Therefore the solution is a stable node for $w<-1$, a saddle point for $-1<w<0$, and an unstable node for $w>0$. Then only the stable fixed point is the class (ii) with $w<-1$. The class (iii) corresponds to the eigenvalues: $\lambda=-3(1+w)$ and $\lambda=-3$. Since $w=-1$ in this case by Eq. (15), one has $\lambda=0,-3$. Therefore the fixed point (iii) is marginally stable.

The above argument corresponds to the one in which $p^{\prime}(\rho)$ asymptotically approaches a constant $w$. We can expect that this argument may be applied to the case with a dynamically changing $p^{\prime}(\rho)$ by following "instantaneous" fixed points as in Refs. 12, 13]. We will check this behavior numerically in Sec. V.

\section{MODELS OF FUTURE SINGULARITIES}

In the present section we will consider the dark energy universe models which contain finite-time, future singularities. It is clear that depending on the content of the model such singularities may behave in different ways. That is why it is useful to classify the future singularities in the following way:

- Type I ("Big Rip") : For $t \rightarrow t_{s}, a \rightarrow \infty, \rho \rightarrow \infty$ and $|p| \rightarrow \infty$

- Type II ("sudden") : For $t \rightarrow t_{s}, a \rightarrow a_{s}, \rho \rightarrow \rho_{s}$ and $|p| \rightarrow \infty$

- Type III : For $t \rightarrow t_{s}, a \rightarrow a_{s}, \rho \rightarrow \infty$ and $|p| \rightarrow \infty$

- Type IV : For $t \rightarrow t_{s}, a \rightarrow a_{s}, \rho \rightarrow 0,|p| \rightarrow 0$ and higher derivatives of $H$ diverge.

Here $t_{s}, a_{s}$ and $\rho_{s}$ are constants with $a_{s} \neq 0$. The type I is so-called the Big Rip singularity [ $[$ ] which emerges for the phantom-like equation of state: $w<-1$. The type II corresponds to the sudden future singularity in [7] at which $a$ and $\rho$ are finite but $p$ diverges. The type III appears for the model with $p=-\rho-A \rho^{\alpha}$ [14], which is different from the sudden future singularity in the sense that $\rho$ diverges. This type of singularity has been discovered in the model of Ref. [6] where the corresponding Lagrangian model of a scalar field with potential has been constructed. The type IV is a new type of singularity which appears in the model described below.

In this section we consider the dark energy EOS characterized by

$$
p=-\rho-f(\rho),
$$

where $f(\rho)$ can be an arbitrary function in general. Note that such EOS maybe equivalent to bulk viscosity, see Refs. [15]. The function $f(\rho) \propto \rho^{\alpha}$ with a constant $\alpha$ was proposed in Ref. [6] and was investigated in detail in Ref. [14]. When $Q=0$ in Eq. (11), the scale factor is given by

$$
a=a_{0} \exp \left(\frac{1}{3} \int \frac{\mathrm{d} \rho}{f(\rho)}\right)
$$

where $a_{0}$ is a constant. Here it is assumed $f(\rho)$ does not vanish for all values of $\rho$. When $f(\rho)=0$ everywhere, the standard $\Lambda \mathrm{CDM}$ cosmology is recovered, so the above choice of EOS is a sound way to study the deviations from such cosmology.

In this section we do not implement the contribution of a barotropic fluid, i.e., $\rho_{m}=0, p_{m}=0$ and $Q=0$ in Eqs. (11)-(4). Combining Eqs. (11) and (41) with (21), one finds

$$
t=\int \frac{\mathrm{d} \rho}{\kappa \sqrt{3 \rho} f(\rho)},
$$

which is used later. Eqs. (3) and (4) give

$$
\frac{\ddot{a}}{a}=-\frac{\kappa^{2}}{6}(\rho+3 p)=\frac{\kappa^{2}}{6}[2 \rho+3 f(\rho)] .
$$

For example, for the specific choice of EOS: $f(\rho)=$ $-2 \rho / 3+\rho_{0} \sin \left(\rho_{1} / \rho\right)$, with constants $\rho_{0}$ and $\rho_{1}$, the universe iterates the transition between the acceleration $(\ddot{a}>0)$ and deceleration $(\ddot{a}<0)$.

\section{A. The model of transition from $w>-1$ to $w<-1$}

In this subsection we study the model in which EOS changes from $w>-1$ to $w<-1$.

When $w$ is a constant, one has $a \sim t^{2 / 3(1+w)}$ and $H \sim 2 / 3(1+w) t$ for $w>-1$, and $a \sim\left(t_{s}-t\right)^{2 / 3(1+w)}$ or $H \sim-2 / 3(1+w)\left(t_{s}-t\right)$ for $w<-1$. It is interesting to understand what kind of $f(\rho)$ admits the transition between the region with $w>-1$ and that with $w<-1$. Let us consider the following simple model

$$
a(t)=a_{0}\left(\frac{t}{t_{s}-t}\right)^{n} .
$$

Here $n$ is a positive constant and $0<t<t_{s}$. The scale factor diverges with a finite time $\left(t \rightarrow t_{s}\right)$ as in 
the Big Rip singularity. Therefore $t_{s}$ corresponds to the life time of the universe. When $t \ll t_{s}, a(t)$ evolves as $t^{n}$, which means that the effective EOS is given by $w=-1+2 /(3 n)>-1$. On the other hand, when $t \sim t_{s}$, it appears $w=-1-2 /(3 n)<-1$.

From Eq. (25) the Hubble rate is given by

$$
H=n\left(\frac{1}{t}+\frac{1}{t_{s}-t}\right) .
$$

Then using Eq. (4), we find

$$
\rho=\frac{3 n^{2}}{\kappa^{2}}\left(\frac{1}{t}+\frac{1}{t_{s}-t}\right)^{2} .
$$

Hence, both $H$ and $\rho$ have minima at $t=t_{s} / 2$ with the values

$$
H_{\min }=\frac{4 n}{t_{s}}, \quad \rho_{\min }=\frac{48 n^{2}}{\kappa^{2} t_{s}^{2}} .
$$

By deleting $t$ in $\dot{\rho}$, one obtains

$$
\dot{\rho}= \pm 2 \rho\left\{\frac{\rho \kappa^{2}}{3 n^{2}}-\frac{4}{n t_{s}}\left(\frac{\kappa^{2} \rho}{3}\right)^{\frac{1}{2}}\right\}^{\frac{1}{2}}
$$

Here the plus sign in Eq. (29) corresponds to the region $t>t_{s} / 2$ and the minus one to the region $t<t_{s} / 2$. Combining Eq. (29) with Eq. (1), it follows

$$
f(\rho)= \pm \frac{2 \rho}{3 n}\left\{1-\frac{4 n}{t_{s}}\left(\frac{3}{\kappa^{2} \rho}\right)^{\frac{1}{2}}\right\}^{\frac{1}{2}}
$$

Therefore the EOS needs to be double-valued in order for the transition to occur between the region $w<-1$ and the region $w>-1$. Such a double-valued EOS often appears when there is a first-order phase transition. As we will see later in Sec. VI, if there are two kinds of matter or energy, a value of $\rho$ can correspond to several values of $p$. We should also note $f\left(\rho_{\text {min }}\right)=0$, that is, the minima of $\rho$ and $H$ correspond to the transition point: $w=-1$.

The singularity at $t=t_{s}$ corresponds to the Big Rip type characterized by $a \rightarrow \infty, \rho \rightarrow \infty$ and $|p| \rightarrow \infty$ for $t \rightarrow t_{s}$. In this region $f(\rho)$ behaves as $f(\rho) \sim 2 \rho / 3 n$ due to Eq. (30), which means that the pressure $p$ is linear in $\rho$, i.e., $p=-\rho-2 \rho / 3 n$. Therefore this gives the constant EOS: $w=\rho / p=-1-2 / 3 n$. In another asymptotic limit $t \rightarrow 0$, one also obtains the constant value of $w$, i.e., $w=-1+2 / 3 n$.

For the general case where $w$ crosses -1 , since $w=$ -1 corresponds to $f(\rho)=0$, in order that the integral $\int \mathrm{d} \rho / f(\rho)$ in Eq. (22) is finite, $f(\rho)$ should behave as

$$
f(\rho) \sim f_{0}\left(\rho-\rho_{0}\right)^{s}, \quad 0<s<1,
$$

where the condition $f\left(\rho_{0}\right)=0$ is assumed. Since $0<s<$ $1, f(\rho)$ should be multi-valued near $\rho=\rho_{0}$ in general.

\section{B. Specific model}

In what follows we shall investigate a model characterized by

$$
f(\rho)=\frac{A B \rho^{\alpha+\beta}}{A \rho^{\alpha}+B \rho^{\beta}},
$$

where $A, B, \alpha$ and $\beta$ are constants. As is shown below, this dark energy scenario contains a rich structure from the viewpoint of singularities.

If $\alpha$ is larger than $\beta$, we have

$$
f(\rho) \rightarrow\left\{\begin{array}{ll}
A \rho^{\alpha} & \text { when } \rho \rightarrow 0 \\
B \rho^{\beta} & \text { when } \rho \rightarrow \infty
\end{array} .\right.
$$

When $\alpha, \beta \neq 1$, Eq. (22) gives

$$
a=a_{0} \exp \left\{-\frac{1}{3}\left[\frac{\rho^{-\alpha+1}}{(\alpha-1) A}+\frac{\rho^{-\beta+1}}{(\beta-1) B}\right]\right\} .
$$

For $1>\alpha>\beta$, if $A, B>0(A, B<0)$, $a$ has a minimum (maximum) $a_{0}$ at $\rho=0$ and $a$ goes to infinity (vanishes) when $\rho \rightarrow \infty$. For $\alpha>1>\beta$, if $A<0$ and $B>0(A>0$ and $B<0$ ), a has a minimum (maximum) at the nontrivial (non-vanishing) value of $\rho$ and $a$ goes to infinity (zero) when $\rho$ vanishes or goes to a positive infinity. For $\alpha>1>\beta$, if $A, B>0(A, B<0), a$ goes to infinity when $\rho \rightarrow \infty(\rho \rightarrow 0)$ and $a$ vanishes when $\rho \rightarrow 0(\rho \rightarrow \infty)$. For $\alpha>\beta>1$, $a$ goes to $a_{0}$ when $\rho \rightarrow \infty$. Furthermore if $A>0(A<0), a \rightarrow 0(a \rightarrow \infty)$ when $\rho \rightarrow 0$. If $A, B>0$ $(A, B<0), a$ is a monotonically increasing (decreasing) function of $\rho$. If $A>0$ and $B<0(A<0$ and $B>0)$, $a$ has a nontrivial maximum (minimum) at a finite value of $\rho$.

\section{The model (32) with $\alpha=2 \beta-1$}

In what follows we shall concentrate on the case that

$$
\alpha=2 \beta-1 \text {. }
$$

Then Eq. (34) may be solved with respect to $\rho$ :

$$
\rho=\left\{-\frac{A}{B} \pm\left(\frac{A^{2}}{B^{2}}-6 A(\beta-1) \ln \frac{a}{a_{0}}\right)^{\frac{1}{2}}\right\}^{-\frac{1}{\beta-1}},
$$

which is valid for $\beta \neq 1$. One has $f(\rho)=A B \rho /(A+B)$ for $\beta=1$, which means that the EOS becomes a usual linear equation $p=w \rho$ with constant $w$, that is, $w=$ $-1-A B /(A+B)$. As this case has been well investigated, we will not consider it in this paper.

When $\alpha=2 \beta-1$, the pressure $p$ is given by

$$
p=-\rho-\frac{A B \rho^{2 \beta-1}}{A \rho^{\beta-1}+B} .
$$


Now Eq. (34) can be rewritten as

$$
a=a_{0} \exp \left\{-\frac{1}{3}\left[\frac{\rho^{-2(\beta-1)}}{2(\beta-1) A}+\frac{\rho^{-(\beta-1)}}{(\beta-1) B}\right]\right\} .
$$

Eq. (38) tells that if $\beta>1, a \rightarrow a_{0}$ when $\rho \rightarrow \infty$, and $a \rightarrow 0(a \rightarrow \infty)$ when $\rho \rightarrow 0$ and $A>0(A<0)$. On the other hand, if $\beta<1, a \rightarrow a_{0}$ when $\rho \rightarrow 0$, and $a \rightarrow 0$ $(a \rightarrow \infty)$ when $\rho \rightarrow \infty$ and $A<0(A>0)$. Furthermore Eq. (37) tells that if $\beta>1$,

$$
\begin{gathered}
p \rightarrow-\rho-A \rho^{2 \beta-1}, \quad \text { when } \quad \rho \rightarrow 0, \\
p \rightarrow-\rho-B \rho^{\beta}, \quad \text { when } \quad \rho \rightarrow \infty .
\end{gathered}
$$

Therefore $w=p / \rho \rightarrow-1-0(-1+0)$ when $\rho \rightarrow 0$ and $A>0(A<0)$ and $w \rightarrow+\infty(-\infty)$ when $\rho \rightarrow \infty$ and $B<0(B>0)$. Thus, except for the case where $\rho \rightarrow 0$ and $A<0$, the dominant energy condition (DEC), which requires,

$$
\rho \geq 0, \quad \rho \pm p \geq 0,
$$

is violated. On the other hand, the strong energy condition (SEC),

$$
\rho+3 p \geq 0, \quad \rho+p \geq 0,
$$

is not violated when $\rho \rightarrow \infty$ and $B<0$. We also note that if $B<0$, the weak energy condition (WEC),

$$
\rho \geq 0, \quad \rho+p \geq 0,
$$

and the null energy condition (NEC),

$$
\rho+p \geq 0,
$$

are not violated, either.

Moreover, if $\beta<1$, one gets

$$
\begin{gathered}
p \rightarrow-\rho-A \rho^{2 \beta-1}, \quad \text { when } \quad \rho \rightarrow \infty, \\
p \rightarrow-\rho-B \rho^{\beta}, \quad \text { when } \quad \rho \rightarrow 0 .
\end{gathered}
$$

Therefore $w \rightarrow+\infty(-\infty)$ when $\rho \rightarrow 0$ and $B<0(B>$ $0)$. Then DEC is violated but if $B<0$, the violation of SEC does not occur. When $\rho \rightarrow \infty$, one has $w \rightarrow-1-0$ $(-1+0)$ for $A>0(A<0)$. Then DEC is not violated if $A<0$ although SEC could be violated. The violation of WEC and NEC does not occur in this case.

From Eq. (37) it follows that $p$ diverges when

$$
\rho=\rho_{s} \equiv\left(-\frac{A}{B}\right)^{-\frac{1}{\beta-1}},
$$

which occurs for $A / B<0$. Then we have $p \rightarrow+\infty(-\infty)$ for $B>0(B<0)$ as $\rho \rightarrow \rho_{s}+0$, and $p \rightarrow+\infty(-\infty)$ for $B<0(B>0)$ as $\rho \rightarrow \rho_{s}-0$. Then when $\rho \rightarrow \rho_{s}+0$ with $B>0$ or $\rho \rightarrow \rho_{s}-0$ with $B<0$, only the violation of DEC occurs, while other energy conditions are not violated.
By integrating Eq. (23), we find

$$
\begin{aligned}
& \frac{2}{4 \beta-3} \rho^{-\frac{4 \beta-3}{2}}+\frac{2 A}{(2 \beta-1) B} \rho^{-\frac{2 \beta-1}{2}} \\
& =-\sqrt{3} \kappa A\left(t-t_{0}\right) \equiv \tau,
\end{aligned}
$$

where $t_{0}$ is an integration constant. This is valid for $\beta \neq 1, \beta \neq 3 / 4$, and $\beta \neq 1 / 2$. When $\beta=3 / 4$, instead of Eq. (48), we obtain

$$
-\ln \frac{\rho}{\rho_{0}}+\frac{4 A}{B \rho^{\frac{1}{4}}}=\tau
$$

where $\rho_{0}$ is a constant introduced for a dimensional reason. For $\beta=1 / 2$, it follows

$$
-2 \rho^{\frac{1}{2}}-\frac{A}{B} \ln \frac{\rho}{\rho_{0}}=\tau .
$$

We use these relations together with Eqs. (36) and (37) in order to study the properties of singularities.

\section{Sudden future singularity for the model (32) with $A / B<0$}

Let us study the property of the singularity at $\rho=$ $\rho_{s}=(-A / B)^{-1 /(\beta-1)}$. This corresponds to a finite value of the scale factor by Eq. (38). When $\rho=\rho_{s}$, Eqs. (48), (49) and (50) give finite values of $\tau$, i.e.,

$$
\tau_{s}=-\left(-\frac{A}{B}\right)^{\frac{4 \beta-3}{2(\beta-1)}} \frac{4(\beta-1)}{(4 \beta-3)(2 \beta-1)},
$$

for $\beta \neq 3 / 4,1 / 2$, and

$$
\tau_{s}=-4\left[\ln \left(-\frac{A}{B \rho_{0}}\right)+1\right],
$$

for $\beta=3 / 4$, and

$$
\tau_{s}=\frac{2 A}{B}\left[1-\ln \left(-\frac{A}{B \rho_{0}}\right)\right],
$$

for $\beta=1 / 2$.

Since $\rho$ is finite, the Friedmann equation (4) also shows that $H$ is finite but from Eq. (24), we find that $\ddot{a}$ diverges because of the divergence of $p$ and the scalar curvature $R$ also diverges since $R=2 \kappa^{2}(\rho-3 p)$. From the above arguments the singularity described by Eq. (47) corresponds to the sudden future singularity (type II) in []].

\section{E. Classification of singularities for the model (32)}

In the previous subsection it has been shown that for all values of $\beta$ the sudden future (type II) singularity appears for the model (32) if $A / B<0$. In addition to this there exists a wide variety of singularities depending on the values of $\beta$. 


\section{1. $\beta>1$}

When $\beta>1 \mathrm{Eq}$. (48) implies that $\rho \rightarrow \infty$ as $\tau \rightarrow+0$ (-0) for $A / B>0(A / B<0)$. From Eqs. (38) and (40) we find $a \rightarrow a_{0}$ and $|p| \rightarrow \infty$ as $\rho \rightarrow \infty$. Therefore this corresponds to the type III singularity at which $\rho$ and $|p|$ diverge with finite $t$ and $a$. The equation of state behaves as $w \rightarrow-1-B \rho^{\beta-1}$ for $\rho \rightarrow \infty$ by Eq. (40), which means that $w \rightarrow+\infty(-\infty)$ for $B<0(B>0)$.

Note that $\tau \rightarrow \infty$ as $\rho \rightarrow 0$ by Eq. (48). For $A / B>0$ the value of $\tau$ is restricted to be $0<\tau<+\infty$. When $A / B<0, \tau$ is restricted to be in the range $\tau>\tau_{s}$ with negative $\tau_{s}$ [see Eq. (51)]. From Eq. (48) we find $\mathrm{d} \tau / \mathrm{d} \rho=$ 0 at $\rho=\rho_{s}$. Then two branches appear for the region of $\rho$, i.e., $0<\rho<\rho_{s}$ corresponding to $\tau_{s}<\tau<+\infty$ and $\rho_{s}<\rho<+\infty$ corresponding to $\tau_{s}<\tau<0$.

$$
\text { 2. } 3 / 4<\beta<1
$$

Let us next consider the case with $3 / 4<\beta<1$. We find $\rho \rightarrow \infty$ as $\tau \rightarrow+0$ from Eq. (48). Eq. (38) tells that $a \rightarrow \infty$ for $A>0$, and Eq. (45) gives $|p| \rightarrow \infty$ for $\rho \rightarrow \infty$. This means that the singularity at $t=t_{0}$ is the type I (Big Rip). Since $w \rightarrow-1-A \rho^{2(\beta-1)}$ for $\rho \rightarrow \infty$ by Eq. 45), we have $w \rightarrow-1+0(-1-0)$ for $A<0$ $(A>0)$.

In the limit $\rho \rightarrow 0$, one has $\tau \rightarrow+\infty(-\infty)$ for $A / B>$ $0(A / B<0)$. The allowed region of $\tau$ is $0<\tau<+\infty$ for $A / B>0$, whereas $\tau<\tau_{s}$ with positive $\tau_{s}$ for $A / B<0$. In the latter case there exist two branches, $0<\rho<$ $\rho_{s}$ corresponding to $-\infty<\tau<\tau_{s}$ and $\rho_{s}<\rho<\infty$ corresponding to $0<\tau<\tau_{s}$.

$$
\text { 3. } 1 / 2 \leq \beta \leq 3 / 4
$$

In the case of $1 / 2<\beta<3 / 4$, Eq. (48) implies that $\rho \rightarrow 0$ as $\tau \rightarrow+\infty(-\infty)$ for $A / B>0(A / B<0)$. Meanwhile we have $\tau \rightarrow-\infty$ as $\rho \rightarrow \infty$. Then the value of $\tau$ is not limited $(-\infty<\tau<+\infty)$ for $A / B>0$. We find $-\infty<\tau<\tau_{s}$ for $A / B<0$, in which case there exist two branches: $0<\rho<\rho_{s}$ and $\rho_{s}<\rho<\infty$. Except for the type II singularity at $\tau=\tau_{s}$ for $A / B<0$, finitetime singularities do not exist for $1 / 2<\beta<3 / 4$. This property also holds for $\beta=3 / 4$ and $\beta=1 / 2$, as can be checked by Eqs. (52) and (53).

$$
\text { 4. } \beta<1 / 2
$$

When $\beta<1 / 2$, one has $\rho \rightarrow \infty$ as $\tau \rightarrow-\infty$ and $\rho \rightarrow 0$ as $\tau \rightarrow-0(+0)$ for $A / B>0(A / B<0)$. Then the range of $\tau$ is $-\infty<\tau<0$ for $A / B>0$, and $0<$ $\tau<\tau_{s}$ corresponding to $0<\rho<\rho_{s}$ and $-\infty<\tau<\tau_{s}$ corresponding to $\rho_{s}<\rho<\infty$ for $A / B<0$.

In the limit $\rho \rightarrow 0$ or $\tau \rightarrow 0$, Eq. (46) tells that $p \rightarrow 0$ for $0<\beta<1 / 2, p \rightarrow-B$ for $\beta=0$, and $p \rightarrow+\infty(-\infty)$ for $\beta<0$ and $B<0(B>0)$. Therefore there exists a type II singularity for $\beta<0\left(a \rightarrow a_{0}, \rho \rightarrow 0,|p| \rightarrow \infty\right.$ as $\left.t \rightarrow t_{0}\right)$. In this case we have $w \rightarrow+\infty(-\infty)$ for $B<0$ $(B>0)$ by Eq. (46).

In the case of $0<\beta<1 / 2$ one has $\ln a / a_{0} \propto \tau^{1-\frac{1}{2 \beta-1}}$. Since $1-1 /(2 \beta-1)>2$ for $0<\beta<1 / 2, H$ and $\dot{H}$ are finite. However $\mathrm{d}^{n} H / \mathrm{d} t^{n}$ diverge for $n>-1 /(2 \beta-1)$ as long as $1-1 /(2 \beta-1)$ is not an integer. This corresponds to the type IV singularity in which higher derivatives of $H$ exhibit divergence even if $a, \rho$ and $p$ are finite as $t \rightarrow t_{0}$. In this case $w \rightarrow+\infty(-\infty)$ for $B<0(B>0)$ by Eq. (46).

When $\beta=0$, we find $a \sim a_{0} \exp \left[B \tau^{2} /\left(12 A^{2}\right)\right]$ for $\rho \sim 0$, showing the absence of the singularity for higher derivatives of $H$. Therefore there is no any future singularity. In this case $w \rightarrow+\infty(-\infty)$ for $B<0(B>0)$ as $\rho \rightarrow 0$.

The obtained results are summarized as follows:

- For $A / B<0$ there is always the type II singularity irrespective of the values of $\beta$.

- Irrelevant to the sign of $A / B$, the types of singularities are different depending on the values of $\beta$.

1. $\beta>1$ :

There is a type III future singularity. DEC is broken. $w \rightarrow+\infty(-\infty)$ for $B<0(B>0)$.

2. $3 / 4<\beta<1$ :

There is a type I future singularity for $A>0$. $\mathrm{DEC}$ is broken for $A>0 . w \rightarrow-1+0(-1-0)$ for $A<0(A>0)$.

3. $1 / 2 \leq \beta \leq 3 / 4$ :

There is no a finite future singularity.

4. $0<\beta<1 / 2$ :

There is a type IV future singularity. $w \rightarrow$ $+\infty(-\infty)$ for $B<0(B>0)$.

5. $\beta=0$ :

There is no finite future singularity, but when $\rho \rightarrow 0, w \rightarrow+\infty(-\infty)$ for $B<0(B>0)$.

6. $\beta<0$ :

There is a type II future singularity. DEC is broken but SEC is not broken for $B<0$. $w \rightarrow+\infty(-\infty)$ for $B<0(B>0)$.

\section{GENERAL STRUCTURE OF THE SINGULARITIES}

In this section we discuss the general structure of singularities. Especially, the relation between the singularities and the behavior of $f(\rho)$ is clarified. 


\section{A. Type I and III singularities}

Let us investigate a situation in which $\rho$ goes to infinity. Then the Hubble rate diverges from the Friedmann equation (4), which leads to the divergence of all curvatures. As in Eq. (33), we assume the form

$$
f(\rho) \rightarrow B \rho^{\beta}, \quad \text { for } \quad \rho \rightarrow \infty .
$$

Here $B$ and $\beta$ are constants. When $\beta=1$, the EOS is a usual linear equation $p=w \rho$ with a constant $w(=$ $-1-B)$.

The model with $f(\rho)=B \rho^{\beta}$ was proposed in Ref. [6] and further investigated in Ref. [14]. Since we are now interested in the structure of the singularities, we assume the behavior (54) with $\beta>0$ only when $\rho$ goes to infinity. Then by using Eq. (22), it follows

$$
a \sim a_{0} \exp \left[\frac{\rho^{1-\beta}}{3(1-\beta) B}\right] .
$$

When $\beta>1$, the scale factor remains finite even if $\rho$ goes to infinity, which corresponds to the type III singularity [14. When $\beta<1$, we find $a \rightarrow \infty(a \rightarrow 0)$ as $\rho \rightarrow \infty$. for $B>0(B<0)$.

Since the pressure is now given by

$$
p \sim-\rho-B \rho^{\beta}
$$

$p$ always diverges when $\rho$ becomes infinite. If $\beta>1$, the EOS parameter $w=p / \rho$ also goes to infinity, that is, $w \rightarrow+\infty(-\infty)$ for $B<0(B>0)$. When $\beta<1$, we have $w \rightarrow-1+0(-1-0)$ for $B<0(B>0)$ as $\rho \rightarrow \infty$.

By using Eq. (23) for the function (54), one finds

$$
t \sim t_{0}+\frac{2}{\sqrt{3} \kappa B} \frac{\rho^{-\beta+1 / 2}}{1-2 \beta}, \quad \text { for } \quad \beta \neq \frac{1}{2},
$$

and

$$
t \sim t_{0}+\frac{\ln \rho}{\sqrt{3} \kappa B}, \quad \text { for } \quad \beta=\frac{1}{2} .
$$

Therefore if $\beta \leq 1 / 2, \rho$ diverges in an infinite future or past. On the other hand, if $\beta>1 / 2$, the divergence of $\rho$ corresponds to a finite future or past.

From the above argument, one can classify the singularities as follows:

1. $\beta>1$ :

There exists a type III singularity. DEC is broken. $w \rightarrow+\infty(-\infty)$ if $B<0(B>0)$.

2. $1 / 2<\beta<1$ :

There is a type I future singularity for $B>0$. DEC is broken in this case. When $B<0$, since $a \rightarrow 0$ as $\rho \rightarrow \infty$, if the singularity exists in past (future), we may call it Big Bang (Big Crunch) singularity. $w \rightarrow-1+0(-1-0)$ if $B<0(B>0)$.
3. $0<\beta \leq 1 / 2$ :

There is no finite future singularity.

When $\beta<0$, it was shown in Ref. 14] that the type II singularity appears as $\rho \rightarrow 0$. In the next subsection we shall investigate a more general model.

\section{B. Type II singularity}

Lets us consider the type II singularity at which $p$ is singular but $\rho$ is nonsingular at a finite time $t=t_{s}$ as in Ref. 7]. The starting function $f(\rho)$ is given by

$$
f(\rho) \sim C\left(\rho_{0}-\rho\right)^{-\gamma},
$$

where $\gamma$ is a positive constant. We concentrate on the case where $\rho$ is smaller than $\rho_{0}$, but the situation is basically the same for $\rho>\rho_{0}$ by considering the function $f(\rho)=C\left(\rho-\rho_{0}\right)^{-\gamma}$. In the limit $\rho \rightarrow \rho_{0}$, the pressure $p$ becomes infinite because of the divergence of $f(\rho)$. The scalar curvature $R$ diverges since $R=2 \kappa^{2}(\rho-3 p)$. The EOS is

$$
w \sim-1-\frac{C}{\rho\left(\rho_{0}-\rho\right)^{\gamma}},
$$

which gives $w \rightarrow-\infty$ for $C>0$ and $w \rightarrow \infty$ for $C<0$ as $\rho \rightarrow \rho_{0}$.

From Eq. (22) the scale factor is integrated to give

$$
a \sim a_{0} \exp \left[-\frac{\left(\rho_{0}-\rho\right)^{\gamma+1}}{3 C(\gamma+1)}\right],
$$

which means that $a$ is finite for $\rho=\rho_{0}$. Since the Hubble rate $H$ is nonsingular by Eq. (4), $\dot{a}$ remains to be finite. On the other hand Eq. (24) implies that $\ddot{a}$ diverges for $\rho \rightarrow \rho_{0}$. By using Eq. (23) we find the following relation around $\rho \sim \rho_{0}$ :

$$
t \sim t_{0}-\frac{\left(\rho_{0}-\rho\right)^{\gamma+1}}{\kappa C \sqrt{3 \rho_{0}}(\gamma+1)},
$$

where $t_{0}$ is an integration constant. Then $t$ is finite $(t=$ $t_{0}$ ) even for $\rho=\rho_{0}$. The above discussion shows that the function $f(\rho)$ in Eq. (59) gives rise to the sudden future singularity.

This type of singularity always appears when the denominator of $f(\rho)$ vanishes at a finite value of $\rho$. The model (54) with negative $\beta$ is a special case of the model (59) with $\rho_{0}=0$.

\section{ATTRACTOR SOLUTIONS}

In this section we account for the contribution of a barotropic perfect fluid and investigate the attractor properties for several models presented in Sec. III. We study the case of a non-relativistic dark matter $\left(w_{m}=0\right)$ without a coupling between dark matter and dark energy. 


\section{A. Constant $p^{\prime}(\rho)$ with $p^{\prime}(\rho) \neq-1$}

Let us first investigate the case in which $p^{\prime}(\rho)$ asymptotically approaches a constant, $p^{\prime}(\rho) \rightarrow w$, with $w \neq-1$. One example is presented in Eq. (30), i.e.,

$$
p=-\rho \pm \frac{2 \rho}{3 n}\left[1-\left(\frac{\rho_{c}}{\rho}\right)^{1 / 2}\right]^{1 / 2}
$$

where $\rho_{c} \equiv 48 n^{2} /\left(\kappa^{2} t_{s}^{2}\right)$. In the limit $\rho \rightarrow \infty$ the minus sign in Eq. (63) gives the constant EOS, $w=$ $-1-2 /(3 n)<-1$, whereas the plus sign gives $w=$ $-1+2 /(3 n)>-1$.

When $-1<w<0$ we showed in Sec. II that the fixed point $(x, y)=[(1+w) / 2,(1-w) / 2]$ is a saddle point. One can easily find that this fixed point is the intersection of the line (16) and the line $x+y=1$. For the model (63) with a plus sign, one has $y / x+1=3 n / \sqrt{1-\left(\rho_{c} / \rho\right)^{1 / 2}}$ by Eq. (15). Since $\sqrt{1-\left(\rho_{c} / \rho\right)^{1 / 2}}<1$, the range of $x$ and $y$ in the phase plane is restricted to be $y>(3 n-1) x$, $y \leq 1-x$ and $x \geq 0$. Note that the line (16) corresponds to $y=(3 n-1) x$ by setting $w=-1+2 /(3 n)$. Therefore the intersection of two lines $y=(3 n-1) x$ and $y=1-x$ is a saddle point given by $(x, y)=[1 /(3 n), 1-1 /(3 n)]$. This is plotted as a point A in Fig. 1]

We run our numerical code for initial conditions of $x$ and $y$ that belong to the allowed range explained above. We find that the solutions gradually approach $x=0$, which gives the EOS: $w=-1$ by Eq. (15). The point A corresponding to $w=-1+2 /(3 n)$ is not an attractor point. As the trajectories approach $x=0$, the energy density $\rho$ decreases to a minimum value $\rho_{c}$. Figure 1 shows that there is some portion of the energy density of a barotropic fluid $\left(\Omega_{m}=1-x-y>0\right)$ around $x=0$ if the initial condition of $x$ is much smaller than $y$. When the trajectories get closer to the line $x+y=1$ in the middle of the way, the final attractor point is $(x, y)=(0,1)$, corresponding to a universe described by a cosmological constant with a vanishing energy density of a barotropic fluid.

When $w<-1$ there exists one stable fixed point: $(x, y)=[(1+w) / 2,(1-w) / 2]$. In scalar-field dark energy models, the kinematic term is negative $(x<0)$ at this critical point. For the model (63) with a minus sign, one can easily show that the allowed range of $x$ and $y$ is described by $y>-(3 n+1) x, y \leq 1-x$ and $x \leq 0$. The intersection of the two lines $y=-(3 n+1) x$ and $y=1-x$, i.e., $(x, y)=[-1 /(3 n), 1+1 /(3 n)]$, represents the above stable fixed point. In Fig. 2 we plot the phase plane in terms of $x$ and $y$ for the model (63) with a minus sign. The solutions approach the above stable fixed point at which the EOS is given by $w=-1-2 /(3 n)<-1$. We recall that this attractor point corresponds to the Big Rip singularity with a divergent energy density at finite time $t_{s}$. If we connect two functions (63) at the minimum value of $\rho$, the solutions starting from the region $x>0$ approach the line $x=0$, enter the region $x<0$,

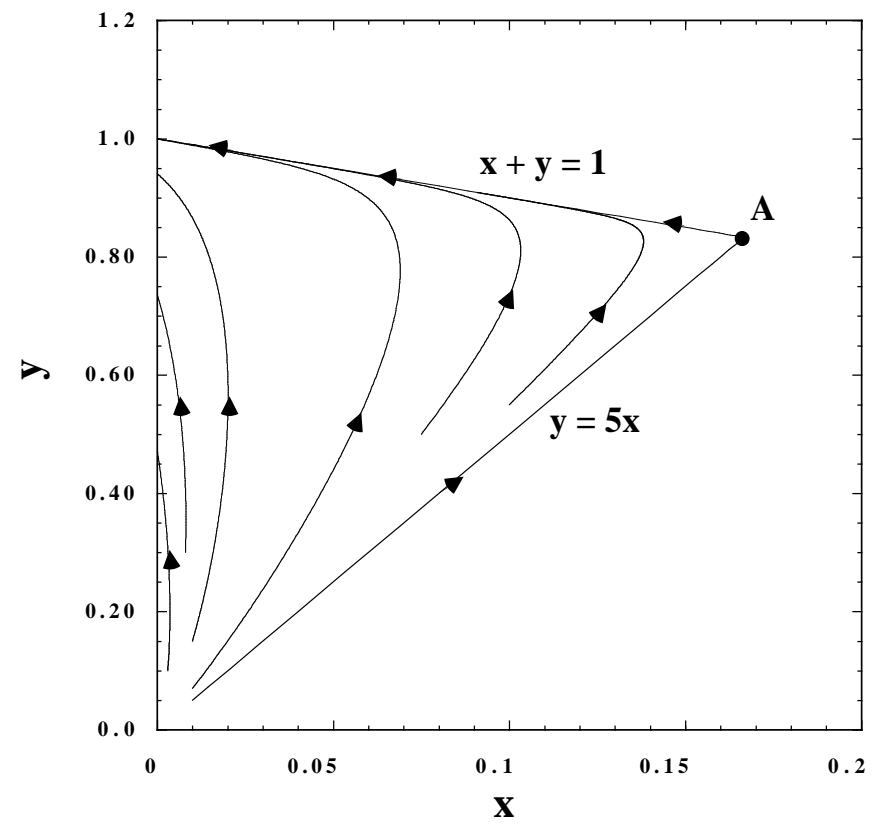

FIG. 1: The phase plane for the plus sign of the model (63) with $n=2$. The allowed range corresponds to $y \leq 1-x$, $y>5 x$ and $x \geq 0$. The point $\mathrm{A}$ is a saddle point given by $x=1 /(3 n)=1 / 6$ and $y=1-1 /(3 n)=5 / 6$. The solutions approach the region $x=0$, corresponding to the equation of state: $w=-1$.

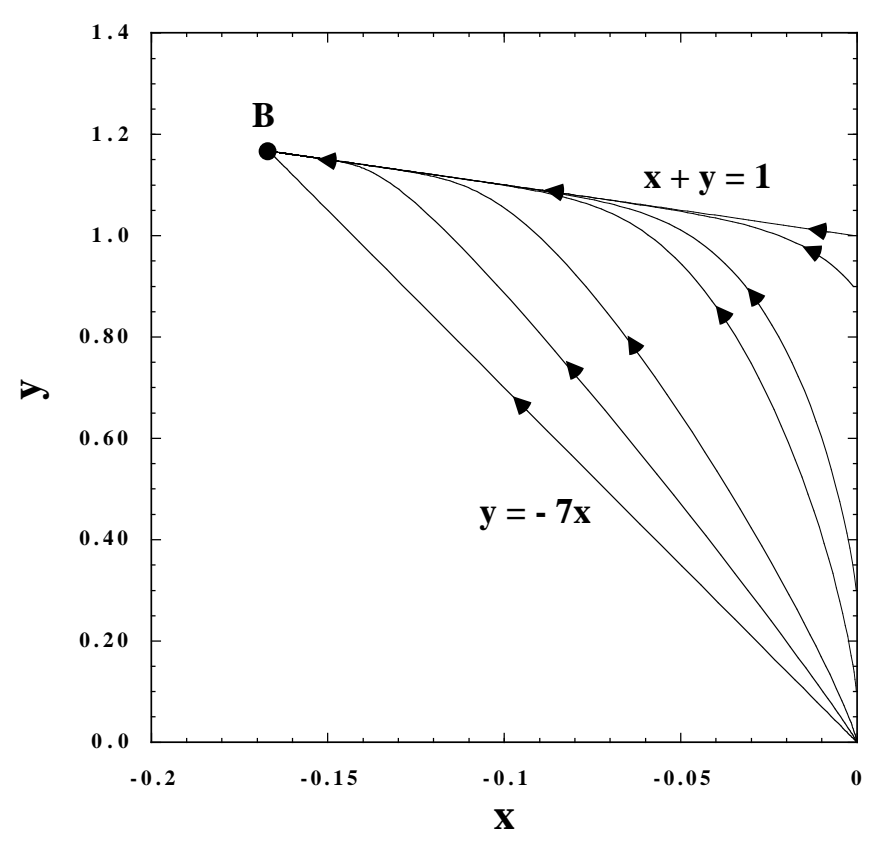

FIG. 2: The phase plane for the minus sign of the model 63) with $n=2$. The allowed range corresponds to $y \leq 1-x$, $y>-7 x$ and $x \leq 0$. The point $\mathrm{B}$ is a stable node given by $x=-1 /(3 n)=-1 / 6$ and $y=1+1 /(3 n)=7 / 6$, which corresponds to the late-time attractor. The equation of state at this point is a constant: $w=-1-2 /(3 n)=-4 / 3$. 
and then approach the above stable fixed point. It is interesting to note that the final attractor point is the Big Rip type even if the system begins from a non-phantom stage $(x>0)$.

\section{B. $\left|p^{\prime}(\rho)\right| \rightarrow \infty$}

Let us next consider the case in which $\left|p^{\prime}(\rho)\right|$ diverges asymptotically. One example is given by the model (37) with $\beta>1$. In this case there exists a type III singularity at which $\rho$ and $p$ exhibit divergence with finite time and scale factor. In the limit $\rho \rightarrow \infty$ the pressure $p$ is given by Eq. (40), which means that $p^{\prime}(\rho) \sim-1-\beta B \rho^{\beta-1} \rightarrow-\infty$ and $w \sim-1-B \rho^{\beta-1} \rightarrow-\infty$ for $B>0$.

We shall study a situation in which the system starts out from the region $x<0$ (i.e., negative $w$ ) and then evolves toward $w \rightarrow-\infty$. By Eqs. (15) and (37) one obtains $y / x=-1-2(A u+B) /\left(A B u^{2}\right)$, where $u \equiv \rho^{\beta-1}>$ 0 . When $A$ and $B$ are both positive, we find $y>-x$ for $x<0$. Therefore the allowed range is restricted to be $y>-x, y \leq 1-x$ and $x<0$ in the phase plane.

In Fig. 3] we plot the trajectories of the solutions for the model (37) with $\beta=1.1, A=2$ and $B=1$. We find that all solutions approach $x \rightarrow-\infty$ and $y \rightarrow+\infty$ along with the line $x+y=1$. The points $(x, y)=$ $\left[\left(1+p^{\prime}(\rho)\right) / 2,\left(1-p^{\prime}(\rho)\right) / 2\right]$ can be regarded as "instantaneous" critical points corresponding to the evolving critical point (ii) introduced in Sec. II. In fact these points are on the line $x+y=1$ and all trajectories approach this line as illustrated in Fig. 3. We note that along the line $x+y=1$ the energy density of the barotropic fluid is vanishing $\left(\Omega_{m}=0\right)$.

\section{C. $p^{\prime}(\rho) \rightarrow-1$}

Finally we consider the situation in which $p^{\prime}(\rho)$ approaches -1 asymptotically. One example is provided by the model (37) with $3 / 4<\beta<1$, in which case there is a type I singularity. Since $p$ is given by Eq. (45) in the limit $\rho \rightarrow \infty$, we have $p^{\prime}(\rho) \sim-1-A(2 \beta-1) \rho^{2(\beta-1)} \rightarrow-1-0$ and $w \sim-1-A \rho^{2(\beta-1)} \rightarrow-1-0$ for $A>0$.

We study the case in which both $A$ and $B$ are positive. When $x<0$ the allowed range of $x$ and $y$ is the same as in the case of the subsection B. We show in Fig. 4 the trajectories of the solutions for $\beta=0.85, A=2$ and $B=$ 1. The solutions eventually approach the point $(x, y)=$ $(0,1)$ along with the line $x+y=1$. This indicates that the trajectories evolve on instantaneous critical points $(x, y)=\left[\left(1+p^{\prime}(\rho)\right) / 2,\left(1-p^{\prime}(\rho)\right) / 2\right]$ with the increase of $p^{\prime}(\rho)$ toward $p^{\prime}(\rho)=-1$. The final state of the universe is dominated by a cosmological constant with vanishing $\Omega_{m}$.

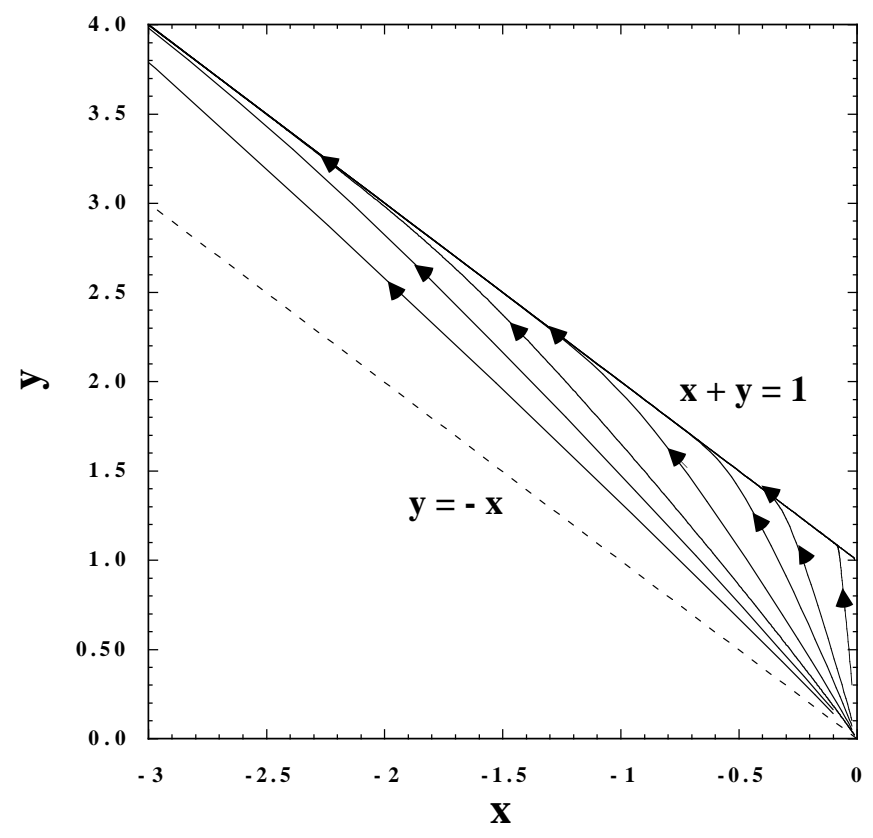

FIG. 3: The phase plane for the model (32) with $\beta=1.1$, $\alpha=2 \beta-1, A=2$ and $B=1$. The solutions approach "instantaneous" critical points: $x=\left(1+p^{\prime}(\rho)\right) / 2$ and $y=$ $\left(1-p^{\prime}(\rho)\right) / 2$, which diverge as $x \rightarrow-\infty$ and $y \rightarrow \infty$ at the type III singularity.

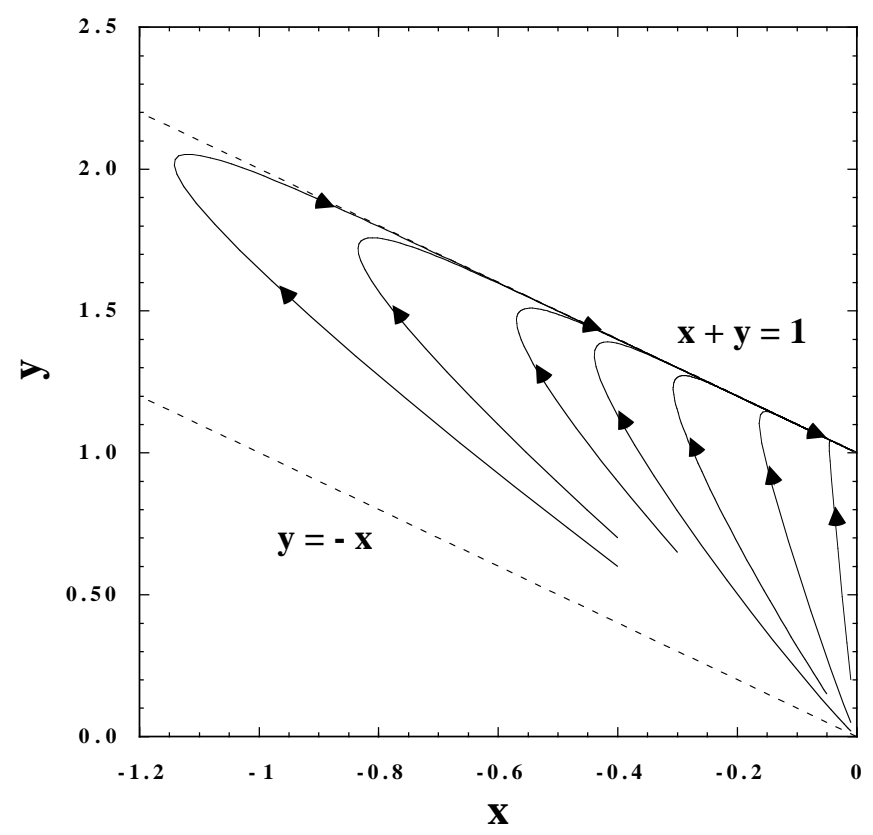

FIG. 4: The phase plane for the model (32) with $\beta=0.85$, $\alpha=2 \beta-1, A=2$ and $B=1$. The solutions approach the type I singularity at which the equation of state is $w=-1$ with $x=0$ and $y=1$. 


\section{COUPLED PHANTOM SCENARIOS}

In this section we shall investigate a situation in which dark energy is coupled to dark matter. The coupled quintessence scenario was originally proposed in Ref. 16] as an extension of nonminimal coupling theories [17]. This scenario has an advantage to explain the coincidence problem, that is, why the energy density of dark energy is comparable with the energy density of dark matter or usual matter. In Ref. [16] the coupling between a scalar field $\phi$ responsible for dark energy and ordinary matter is assumed to take the forms: $T_{\nu(\phi) ; \mu}^{\mu}=C T_{(m)} \phi_{; \nu}$ and $T_{\nu(m) ; \mu}^{\mu}=-C T_{(m)} \phi_{; \nu}$, where $T_{\mu \nu(\phi)}$ and $T_{\mu \nu(m)}$ are the energy momentum tensors of $\phi$ and ordinary matter, respectively. Recently, a system of a phantom scalar field coupled to dark matter was studied in Ref. [18] by writing the FLRW equations in an autonomous form, but this is not equivalent to our analysis since we assumed the EOS of dark energy rather than the existence of a scalar field. Furthermore the forms of the coupling $Q$ are different from the ones we consider in this paper.

\section{A. Analytic solutions for coupled phantom}

Let the dark energy be a fluid whose equation of motion is given by Eq. (11). In general the coupling $Q$ can be the function of $a, H, \rho_{m}, \dot{\rho}_{m}, \rho$ and $\dot{\rho}$. We can express the coupling $Q$ that corresponds to scaling solutions, that is, the ratio $r=\rho_{m} / \rho$ is a constant. By Eqs. (1) and (2) we find

$$
\dot{r}=r\left[\frac{Q}{\rho_{m}}+\frac{Q}{\rho}-3 H\left(w_{m}-w\right)\right] .
$$

We obtain the coupling $Q$ for the existence of scaling solutions [19, 20], as

$$
Q=3 H\left(w_{m}-w\right) \frac{\rho \rho_{m}}{\rho+\rho_{m}}
$$

which is derived by setting $\dot{r}=0$. In this case the energy density of ordinary matter does not vanish asymptotically.

Several authors considered the coupling of the forms like $Q=3 H \delta\left(\rho+\rho_{m}\right)$ [20] and $Q=\delta H \rho_{m}$ [21], where $\delta$ is a coupling strength. In this work we are interested in the coupling with which singularities discussed in preceding sections can be obtained analytically.

Let us consider the following coupling as a toy model:

$$
Q=\delta H^{2}
$$

where $\delta$ is a constant. As usually dark matter is regarded as a dust, i.e.,

$$
p_{m}=0 \text {. }
$$

The following EOS for dark energy is assumed:

$$
p=-2 \rho,
$$

that is, $f(\rho)=\rho$ in Eq. (21).

Then combining Eq. (4) with Eqs. (1) and (2), we find a solution

$$
\begin{aligned}
H & =\frac{2}{3}\left(\frac{1}{t}+\frac{1}{t_{s}-t}\right) \\
\rho_{m} & =\frac{4}{3 \kappa^{2}}\left(\frac{1}{t}+\frac{1}{t_{s}-t}\right) \frac{1}{t}, \\
\rho & =\frac{4}{3 \kappa^{2}}\left(\frac{1}{t}+\frac{1}{t_{s}-t}\right) \frac{1}{t_{s}-t},
\end{aligned}
$$

where

$$
t_{s} \equiv \frac{9}{\delta \kappa^{2}} .
$$

The same solution can be obtained if, instead of Eq. (66), the following coupling is adopted

$$
Q=\frac{9 H \rho \rho_{m}}{2\left(\rho+\rho_{m}\right)} .
$$

In this case $t_{s}$ appears as an undetermined constant. The solution (69) is the same as Eq. (26) with the choice $n=$ $2 / 3$. Then the above solution gives rise to the Big Rip singularity at $t=t_{s}$.

In the absence of the coupling $Q$, it is rather difficult to realize a model in which the equation of state $w$ changes from $w>-1$ to $w<-1$ unless the function $f(\rho)$ is double-valued as discussed in Sec. III. However this can be realized if the phantom is coupled to dark matter with an appropriate coupling. We note that the ratio $r=$ $\rho_{m} / \rho$ behaves as $r=\left(t_{s}-t\right) / t$ by Eqs. (70) and (71), which means that this ratio is dynamically changing and vanishes at $t=t_{s}$. Therefore the solution (69) does not correspond to scaling solutions. This is understandable, since the system is completely dominated by the energy density of dark energy around the Big Rip.

\section{B. Multiple scalar field model}

It is possible to construct a scalar-field model which reproduces the solutions (69)-(71). This can be realized by a four-dimensional Lagrangian with two scalar fields $\phi$ and $\chi$ :

$$
\mathcal{L}=\frac{1}{2 \kappa^{2}} R-\frac{1}{2}(\nabla \phi)^{2}+\frac{1}{2}(\nabla \chi)^{2}-V(\phi, \chi),
$$

where the effective potential is

$$
V(\phi, \chi)=\frac{\eta^{2}}{t_{0}^{2}} \mathrm{e}^{-\frac{\phi+\chi}{\phi_{0}}}+\frac{\eta^{2}-\phi_{0}^{2}}{2 t_{0}^{2}} \mathrm{e}^{-\frac{2 \phi}{\phi_{0}}}+\frac{\eta^{2}+\phi_{0}^{2}}{2 t_{0}^{2}} \mathrm{e}^{-\frac{2 \chi}{\phi_{0}}}
$$

Here $\phi_{0}$ and $t_{0}$ are constants, and

$$
\eta^{2}=\frac{3}{2} \kappa^{2} \phi_{0}^{4}
$$


In Eq. (74) the sign of the kinetic term of $\chi$ is opposite compared to the canonical one, which tells that the field $\chi$ is regarded as a phantom. Then in this model, if we consider the quantization, the problem of negative norm states would occur as the phantom is ghost [22, 23, 24]. When we adopt the Brans-Dicke type theory as in Ref. [4], it is possible to avoid the problem of the negative sign in the kinetic term. Since it makes, however, all things complicated, we pay our attention to the scenario given by (74) in order to show how the solutions (69)-(71) are realized in a simple scalar field model.

The total energy density $\rho_{t}$ corresponding to the Lagrangian (74) is given by

$$
\begin{aligned}
\rho_{t}= & \frac{1}{2} \dot{\phi}^{2}-\frac{1}{2} \dot{\chi}^{2}+\frac{\eta^{2}}{t_{0}^{2}} \mathrm{e}^{-\frac{\phi+\chi}{\phi_{0}}} \\
& +\frac{\eta^{2}-\phi_{0}^{2}}{2 t_{0}^{2}} \mathrm{e}^{-\frac{2 \phi}{\phi_{0}}}+\frac{\eta^{2}+\phi_{0}^{2}}{2 t_{0}^{2}} \mathrm{e}^{-\frac{2 \chi}{\phi_{0}}} .
\end{aligned}
$$

In the FRW background the equations of motion for scalar fields are

$$
\begin{aligned}
& \ddot{\phi}+3 H \dot{\phi}-\frac{\eta^{2}}{\phi_{0} t_{0}^{2}} \mathrm{e}^{-\frac{\phi+\chi}{\phi_{0}}}-\frac{\eta^{2}-\phi_{0}^{2}}{\phi_{0} t_{0}^{2}} \mathrm{e}^{-\frac{2 \phi}{\phi_{0}}}=0, \\
& \ddot{\chi}+3 H \dot{\chi}+\frac{\eta^{2}}{\phi_{0} t_{0}^{2}} \mathrm{e}^{-\frac{\phi+\chi}{\phi_{0}}}+\frac{\eta^{2}+\phi_{0}^{2}}{\phi_{0} t_{0}^{2}} \mathrm{e}^{-\frac{2 \chi}{\phi_{0}}}=0 .
\end{aligned}
$$

Then by using the Friedmann equation $H^{2}=\kappa^{2} \rho_{t} / 3$, we find a solution:

$$
\begin{aligned}
& H=\frac{\kappa^{2} \phi_{0}^{2}}{2}\left(\frac{1}{t}+\frac{1}{t_{s}-t}\right) \\
& \phi=\phi_{0} \ln \frac{t}{t_{0}}, \quad \chi=\phi_{0} \ln \frac{t_{s}-t}{t_{0}}
\end{aligned}
$$

where $t_{s}$ appears as a constant of the integration. Then if one chooses $\phi_{0}$ as

$$
\phi_{0}^{2}=\frac{4}{3 \kappa^{2}}
$$

the Hubble rate $H$ in Eq. 69 is reproduced.

The field $\phi$ is identified as dark matter with an energy density $\rho_{m}$ and the field $\chi$ as dark energy with an energy density $\rho$. The total energy density $\rho_{t}$ in Eq. (77) may be given by

$$
\begin{aligned}
\rho_{m} & =\frac{1}{2} \dot{\phi}^{2}+\frac{\eta^{2}}{2 t_{0}^{2}} \mathrm{e}^{-\frac{\phi+\chi}{\phi_{0}}}+\frac{\eta^{2}-\phi_{0}^{2}}{2 t_{0}^{2}} \mathrm{e}^{-\frac{2 \phi}{\phi_{0}}} \\
& =\frac{\eta^{2}}{2}\left(\frac{1}{t}+\frac{1}{t_{s}-t}\right) \frac{1}{t}, \\
\rho & =-\frac{1}{2} \dot{\chi}^{2}+\frac{\eta^{2}}{2 t_{0}^{2}} \mathrm{e}^{-\frac{\phi+\chi}{\phi_{0}}}+\frac{\eta^{2}+\phi_{0}^{2}}{2 t_{0}^{2}} \mathrm{e}^{-\frac{2 \chi}{\phi_{0}}} \\
& =\frac{\eta^{2}}{2}\left(\frac{1}{t}+\frac{1}{t_{s}-t}\right) \frac{1}{t_{s}-t},
\end{aligned}
$$

which agree with Eqs. (70) and (71) by using Eqs. (76) and (82). Furthermore if we define the pressures $p_{m}$ and $p$ as

$$
\begin{aligned}
p_{m} & =\frac{1}{2} \dot{\phi}^{2}-\frac{\eta^{2}}{2 t_{0}^{2}} \mathrm{e}^{-\frac{\phi+\chi}{\phi_{0}}}-\frac{\eta^{2}-\phi_{0}^{2}}{2 t_{0}^{2}} \mathrm{e}^{-\frac{2 \phi}{\phi_{0}}} \\
p & =-\frac{1}{2} \dot{\chi}^{2}-\frac{\eta^{2}}{2 t_{0}^{2}} \mathrm{e}^{-\frac{\phi+\chi}{\phi_{0}}}-\frac{\eta^{2}+\phi_{0}^{2}}{2 t_{0}^{2}} \mathrm{e}^{-\frac{2 \chi}{\phi_{0}}}
\end{aligned}
$$

it follows

$$
\begin{aligned}
& \dot{\rho}_{m}+3 H\left(\rho_{m}+p_{m}\right)=-\{\dot{\rho}+3 H(\rho+p)\} \\
& =\frac{\eta^{2}}{2 \phi_{0} t_{0}^{2}} \mathrm{e}^{-\frac{\phi+\chi}{\phi_{0}}}(\dot{\phi}-\dot{\chi}) .
\end{aligned}
$$

By comparing Eq. (85) with Eqs. (11) and (2), one gets

$$
Q=\frac{\eta^{2}}{2 \phi_{0} t_{0}^{2}} \mathrm{e}^{-\frac{\phi+\chi}{\phi_{0}}}(\dot{\phi}-\dot{\chi}) \text {. }
$$

Note that all the parameters in the action (74) have the scale of order the Planck mass. The parameter $t_{s}$ appears dynamically as a constant of the integration. If the present universe corresponds to the time $t$ of order $t_{s}$, one can estimate $t_{s}$ as $t_{s} \sim 1.8 \times 10^{10}$ years $\sim\left(10^{-33} \mathrm{eV}\right)^{-1}$. The coupling $Q$ in Eq. (86) cannot be straightly rewritten in the form of Eq. (66) or Eq. (73), but with the solution, all the expressions for $H, \rho_{m}$ and $\rho$ give the same functions in terms of the time $t$.

One can consider a generalization of the model in Eq. (74) as follows:

$$
\begin{aligned}
\mathcal{L}= & \frac{1}{2 \kappa^{2}} R-\frac{1}{2}(\nabla \phi)^{2}+\frac{1}{2}(\nabla \chi)^{2}-\frac{\eta \theta}{t_{0}^{2}} \mathrm{e}^{-\frac{\phi}{\phi_{0}}-\frac{\chi}{\zeta_{0}}} \\
& -\frac{\eta^{2}-\phi_{0}^{2}}{2 t_{0}^{2}} \mathrm{e}^{-\frac{2 \phi}{\phi_{0}}}-\frac{\theta^{2}+\chi_{0}^{2}}{2 t_{0}^{2}} \mathrm{e}^{-\frac{2 \chi}{\chi_{0}}} .
\end{aligned}
$$

Here $\phi_{0}, \chi_{0}$, and $t_{0}$ are constants and

$$
\eta^{2}=\frac{3}{2} \kappa^{2} \phi_{0}^{4}, \quad \theta^{2}=\frac{3}{2} \kappa^{2} \chi_{0}^{4} .
$$

Then a solution has the following form:

$$
\begin{aligned}
& H=\frac{\kappa^{2}}{2}\left(\frac{\phi_{0}^{2}}{t}+\frac{\chi_{0}^{2}}{t_{s}-t}\right), \\
& \phi=\phi_{0} \ln \frac{t}{t_{0}}, \quad \chi=\chi_{0} \ln \frac{t_{s}-t}{t_{0}} .
\end{aligned}
$$

The Hubble parameter has a minimum at $t=\phi_{0} t_{s} /\left(\phi_{0}+\right.$ $\left.\chi_{0}\right)$ for $\chi_{0} / \phi_{0}>0$ and diverges for $t \rightarrow 0$ and $t \rightarrow t_{s}$. When $t \ll t_{s}$ the system is dominated by the field $\phi$ and the effective EOS is given by $w \sim-1+4 /\left(3 \kappa^{2} \phi_{0}^{2}\right)$. On the other hand the field $\chi$ dominates around $t \sim$ $t_{s}$, corresponding to the equation of state: $w \sim-1-$ $4 /\left(3 \kappa^{2} \chi_{0}^{2}\right)$. Then the system transits from the region $w>-1$ to the region $w<-1$ and approaches the Big Rip singularity at $t=t_{s}$.

We may investigate whether the present model can satisfy the present observational data. The deceleration of 
the universe changed to the acceleration about five billion years ago. Let us write the corresponding time as $t=t_{c}$. Since

$$
\begin{aligned}
\ddot{a} / a= & H^{2}+\dot{H} \\
= & \frac{\kappa^{2}}{4}\left\{\frac{\left(\kappa^{2} \phi_{0}^{2}-2\right) \phi_{0}^{2}}{t^{2}}+\frac{\left(\kappa^{2} \chi_{0}^{2}+2\right) \chi_{0}^{2}}{\left(t_{s}-t\right)^{2}}\right. \\
& \left.+\frac{2 \kappa^{2} \phi_{0}^{2} \chi_{0}^{2}}{t\left(t_{s}-t\right)}\right\},
\end{aligned}
$$

then $t_{c}$ satisfies

$$
\begin{aligned}
& \left(\phi_{0}^{2}-\chi_{0}^{2}\right)\left\{\kappa^{2}\left(\phi_{0}^{2}-\chi_{0}^{2}\right)-2\right\} t_{c}^{2} \\
& -2 \phi_{0}^{2}\left\{\kappa^{2}\left(\phi_{0}^{2}-\chi_{0}^{2}\right)-2\right\} t_{s} t_{c}+\left(\kappa^{2} \phi_{0}^{2}-2\right) \phi_{0}^{2} t_{s}^{2}=0 .
\end{aligned}
$$

According to the present data, the density of dark energy is $72 \%$ of the total density and that of dark matter is $21 \%$ [25]. We define the energy density of dark matter $\rho_{m}$ and that of the phantom $\rho$ by

$$
\begin{aligned}
\rho_{m} & =\frac{1}{2} \dot{\phi}^{2}+\frac{\eta \theta}{2 t_{0}^{2}} \mathrm{e}^{-\frac{\phi}{\phi_{0}}-\frac{\chi}{\chi_{0}}}+\frac{\eta^{2}-\phi_{0}^{2}}{2 t_{0}^{2}} \mathrm{e}^{-2 \frac{\phi}{\phi_{0}}} \\
& =\frac{3 \kappa^{2} \phi_{0}^{2}}{4 t}\left(\frac{\phi_{0}^{2}}{t}+\frac{\chi_{0}^{2}}{t_{s}-t}\right) \\
\rho & =-\frac{1}{2} \dot{\chi}^{2}+\frac{\eta \theta}{2 t_{0}^{2}} \mathrm{e}^{-\frac{\phi}{\phi_{0}}-\frac{\chi}{\chi_{0}}}+\frac{\theta^{2}+\chi_{0}^{2}}{2 t_{0}^{2}} \mathrm{e}^{-2 \frac{\chi}{\chi_{0}}} \\
& =\frac{3 \kappa^{2} \chi_{0}^{2}}{4\left(t_{s}-t\right)}\left(\frac{\phi_{0}^{2}}{t}+\frac{\chi_{0}^{2}}{t_{s}-t}\right) .
\end{aligned}
$$

If $t$ corresponds to the present age of the universe, that is, fourteen billion years, one finds

$$
\frac{t \chi_{0}^{2}}{\left(t_{s}-t\right) \phi_{0}^{2}} \sim \frac{7}{2}
$$

Then

$$
\begin{aligned}
t & \sim\left(\frac{2 \chi_{0}^{2}}{7 \phi_{0}^{2}}+1\right)^{-1} t_{s} \\
t_{c} & \sim \frac{14-5}{14} t \sim \frac{9}{14}\left(\frac{2 \chi_{0}^{2}}{7 \phi_{0}^{2}}+1\right)^{-1} t_{s} .
\end{aligned}
$$

If we define a coincidence time $\hat{t}$ by $\rho=\rho_{m}$, one has

$$
\hat{t}=\left(1+\frac{\chi_{0}^{2}}{\phi_{0}^{2}}\right) \text {. }
$$

Dark matter is regarded as a nonrelativistic matter with EOS: $w_{m}=0$. We may consider a situation in which this originates from the field $\phi$ with constant EOS: $w_{m}=-1+4 /\left(3 \kappa^{2} \phi_{0}^{2}\right)$, which gives $\phi_{0}^{2}=4 /\left(3 \kappa^{2}\right)$. We caution that this property does not necessarily hold, since this constant EOS does not correspond to the attractor solution as shown in Sec. V. We just wish to estimate the approximate time $t_{s}$ by imposing this condition. Then substituting $\phi_{0}^{2}=4 /\left(3 \kappa^{2}\right)$ and Eq. (96) for Eq. (92), we obtain

$$
\kappa^{2} \chi_{0}^{2}=0.0831097 \ldots
$$

This gives $t_{s}-t \sim 0.02 t \sim 0.3$ billion years toward the Big Rip, which is rather a short time. The coincidence time $\hat{t}$ is given by $t-\hat{t} \sim 0.04 t \sim 0.6$ billion years ago.

\section{ACCOUNT OF QUANTUM EFFECTS AROUND THE SINGULARITIES}

It is quite natural to investigate the role of quantum effects near singularities. Indeed, the dark energy universe with a singularity typically evolves to its end with the growth of the energy. As a consequence the curvature of the universe grows as well, which implies the beginning of a second quantum gravity era. The type IV singularity introduced in Sec. III is not of this sort because the curvature does not grow significantly there.

In what follows we shall study the effect of quantum backreaction of conformal matter around the type I (Big Rip), type II (sudden future) and type III singularities. In these cases, the curvature of the universe becomes large around the singularity at $t=t_{s}$, although the scale factor $a$ is finite for type II and III singularities. Since quantum corrections usually contain the powers of the curvature or higher derivative terms, such correction terms play important roles near the singularity. We now include the quantum effects by taking into account the contribution of conformal anomaly as a backreaction. The conformal anomaly $T_{A}$ has the following form:

$$
T_{A}=b\left(F+\frac{2}{3} \square R\right)+b^{\prime} G+b^{\prime \prime} \square R,
$$

where $F$ is the square of a $4 \mathrm{~d}$ Weyl tensor, $G$ is a GaussBonnet curvature invariant, which are given as

$$
\begin{aligned}
& F=(1 / 3) R^{2}-2 R_{i j} R^{i j}+R_{i j k l} R^{i j k l}, \\
& G=R^{2}-4 R_{i j} R^{i j}+R_{i j k l} R^{i j k l} .
\end{aligned}
$$

In general, with $N$ scalar, $N_{1 / 2}$ spinor, $N_{1}$ vector fields, $N_{2}$ (=0 or 1) gravitons and $N_{\mathrm{HD}}$ higher derivative conformal scalars, the coefficients $b$ and $b^{\prime}$ are given by

$$
\begin{aligned}
& b=\frac{N+6 N_{1 / 2}+12 N_{1}+611 N_{2}-8 N_{\mathrm{HD}}}{120(4 \pi)^{2}}, \\
& b^{\prime}=-\frac{N+11 N_{1 / 2}+62 N_{1}+1411 N_{2}-28 N_{\mathrm{HD}}}{360(4 \pi)^{2}} .
\end{aligned}
$$

We have $b>0$ and $b^{\prime}<0$ for the usual matter except for higher derivative conformal scalars. Notice that $b^{\prime \prime}$ can be shifted by a finite renormalization of the local counterterm $R^{2}$, so $b^{\prime \prime}$ can be arbitrary.

In terms of the corresponding energy density $\rho_{A}$ and the pressure density $p_{A}, T_{A}$ is given by $T_{A}=-\rho_{A}+3 p_{A}$. Using the energy conservation law in FLRW universe:

$$
\dot{\rho}_{A}+3 H\left(\rho_{A}+p_{A}\right)=0,
$$


we may delete $p_{A}$ as

$$
T_{A}=-4 \rho_{A}-\dot{\rho}_{A} / H .
$$

This gives the following expression for $\rho_{A}$ :

$$
\begin{aligned}
\rho_{A}= & -\frac{1}{a^{4}} \int \mathrm{d} t a^{4} H T_{A} \\
= & -\frac{1}{a^{4}} \int \mathrm{d} t a^{4} H\left[-12 b \dot{H}^{2}+24 b^{\prime}\left(-\dot{H}^{2}+H^{2} \dot{H}+H^{4}\right)\right. \\
& \left.-\left(4 b+6 b^{\prime \prime}\right)\left(\dddot{H}+7 H \ddot{H}+4 \dot{H}^{2}+12 H^{2} \dot{H}\right)\right] \cdot(104)
\end{aligned}
$$

A different form of $\rho_{A}$ was obtained in Ref. [26] by requiring that the quantum corrected energy momentum tensor $T_{A \mu \nu}$ has the form as $T_{A \mu \nu}=\left(T_{A} / 4\right) g_{\mu \nu}$ in the conformal metric case rather than assuming the conservation law (102). The quantum corrected FLRW equation is

$$
\frac{3}{\kappa^{2}} H^{2}=\rho+\rho_{A}
$$

Since the curvature is large around $t=t_{s}$, one may assume $\left(3 / \kappa^{2}\right) H^{2} \ll\left|\rho_{A}\right|$. Then $\rho \sim-\rho_{A}$ from Eq. (104), which gives

$$
\begin{aligned}
& \dot{\rho}+4 H \rho \\
& =H\left[-12 b \dot{H}^{2}+24 b^{\prime}\left(-\dot{H}^{2}+H^{2} \dot{H}+H^{4}\right)\right. \\
& \left.-\left(4 b+6 b^{\prime \prime}\right)\left(\dddot{H}+7 H \ddot{H}+4 \dot{H}^{2}+12 H^{2} \dot{H}\right)\right] .
\end{aligned}
$$

From the energy conservation law $\dot{\rho}+3 H(\rho+p)=0$ for $p=-\rho-f(\rho)$, one obtains

$$
H=\frac{\dot{\rho}}{3 f(\rho)} .
$$

\section{A. Type III singularity}

For the type III singularity, the energy density $\rho$ diverges at $t=t_{s}$. Let us consider the function $f(\rho) \sim B \rho^{\beta}$ discussed in Sec. IV. In the absence of quantum corrections the type III singularity appears for $\beta>1$ and $\rho$ behaves as $\rho \propto\left(t_{s}-t\right)^{\frac{2}{1-2 \beta}}$. When quantum corrections are taken into account, it is natural to assume that near the singularity $\rho$ behaves as

$$
\rho=\rho_{0}\left(t_{s}-t\right)^{\tilde{\gamma}} .
$$

As $\rho$ may diverge at $t=t_{s}$, we consider negative values of $\tilde{\gamma}$. Using Eq. (107), one finds

$$
H=-\frac{\tilde{\gamma} \rho_{0}^{1-\beta}}{3 B}\left(t_{s}-t\right)^{-1+\tilde{\gamma}(1-\beta)} .
$$

Since we are considering the case $\beta>1$ and $\tilde{\gamma}<0$, we have that $\tilde{\gamma}(1-\beta)>0$. By picking up most singular term in the r.h.s of Eq. (106), it follows

$$
\dot{\rho} \sim-6\left(\frac{2}{3} b+b^{\prime \prime}\right) H \dddot{H}
$$

Then substituting Eqs. (108) and (109) for Eq. (110), we obtain

$$
\tilde{\gamma}=\frac{4}{1-2 \beta} .
$$

This means that $\rho$ and $H$ evolve as

$$
\rho \propto\left(t_{s}-t\right)^{\frac{4}{1-2 \beta}}, \quad H \propto\left(t_{s}-t\right)^{\frac{3-2 \beta}{1-2 \beta}},
$$

around $t=t_{s}$. Compared with the classical evolution: $\rho \propto\left(t_{s}-t\right)^{\frac{2}{1-2 \beta}}$, the energy density diverges more rapidly because of the condition: $4 /(1-2 \beta)<$ $2 /(1-2 \beta)<0$ for $\beta>1$. On the other hand the Hubble rate behaves as $H \propto\left(t_{s}-t\right)^{\frac{1}{1-2 \beta}}$ in the classical case, which means that $H$ is less singular in the presence of quantum corrections because of the condition: $(3-2 \beta) /(1-2 \beta)>1 /(1-2 \beta)$ for $\beta>1$.

We numerically solve the background equations and show the evolution of the Hubble rate for $\beta=2$ and $B>0$ in Fig. 5 In the presence of quantum corrections one has $H \propto\left(t_{s}-t\right)^{1 / 3}$ around $t=t_{s}$, which means that $H$ approaches zero. Meanwhile in the absence of quantum corrections we have $H \propto\left(t_{s}-t\right)^{-1 / 3}$, thereby showing the divergence of $H$ at $t=t_{s}$. These properties are clearly seen in Fig. 5

From Eq. 109 we obtain

$$
a \sim a_{0} \exp \left[\frac{\rho_{0}^{1-\beta}}{3 B(1-\beta)}\left(t_{s}-t\right)^{\tilde{\gamma}(1-\beta)}\right],
$$

where $a_{0}$ is a constant. Comparing the classical case $[\tilde{\gamma}=$ $2 /(1-2 \beta)]$ with the quantum corrected one $[\tilde{\gamma}=4 /(1-$ $2 \beta)]$, we find that the power of $\left(t_{s}-t\right)$ is larger in the presence of quantum corrections. Then the scale factor approaches a constant $a_{0}$ more rapidly if we account for the quantum effect, implying that the spacetime tends to be smooth, although the divergence of $\rho$ is stronger. Thus quantum effects moderate the classical singularity.

\section{B. Type I singularity}

We shall next consider the model $f(\rho) \sim B \rho^{\beta}$ with $1 / 2<\beta<1$ when $\rho$ is large. In this case there exists the Big Rip singularity as shown in Sec. IV. We note that the classical evolution is characterized by $\rho \propto\left(t_{s}-t\right)^{\frac{2}{1-2 \beta}}$ and $H \propto\left(t_{s}-t\right)^{\frac{1}{1-2 \beta}}$, both of which exhibit divergence for $\beta>1 / 2$.

When the quantum correction is present, let us first assume that the time-dependence of $\rho$ is given by Eq. (108) with negative $\tilde{\gamma}$. Since $\tilde{\gamma}(1-\beta)<0$ in this case, we might expect that Eq. (106) would give the following approximate relation around $t=t_{s}$ :

$$
\rho \sim 6 b^{\prime} H^{4} .
$$

The term on the r.h.s. grows as $H^{4} \propto\left(t_{s}-t\right)^{-4+4 \tilde{\gamma}(1-\beta)}$, but this does not give a consistent result, since $\rho$ becomes negative for $b^{\prime}<0$. 


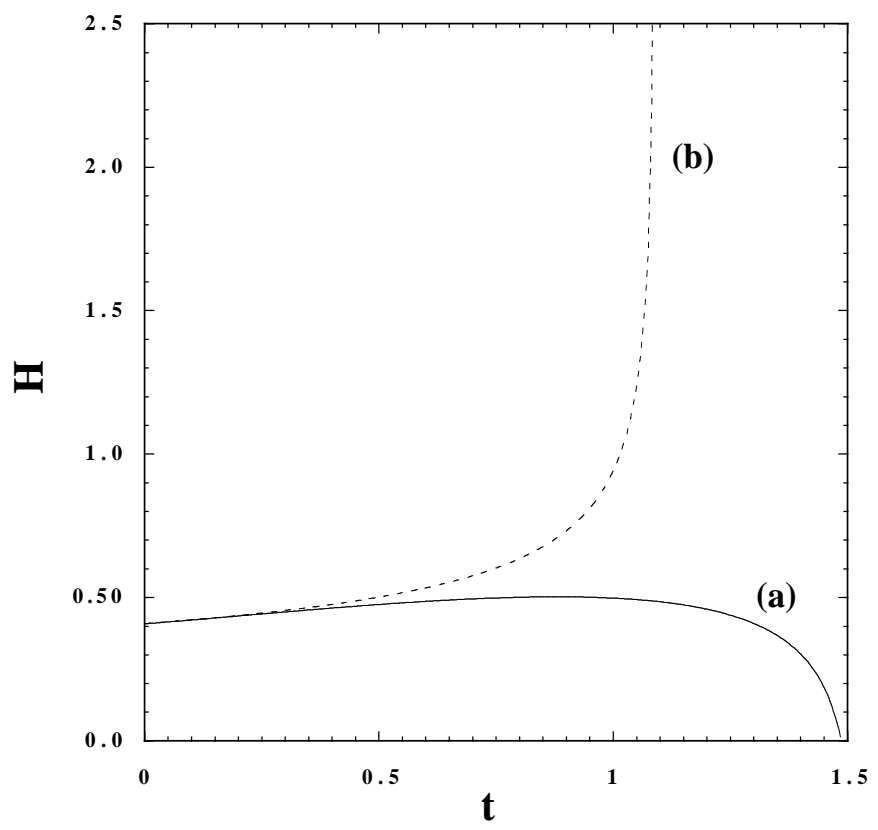

FIG. 5: The evolution of $H$ for the model $f(\rho)=B \rho^{\beta}$ with $\beta=2$ and $B>0$. The case (a) corresponds to the one in which quantum corrections are taken into account with coefficients $b=0.5, b^{\prime}=-0.1$, and $b^{\prime \prime}=0$, whereas the case (b) does not implement such effects. The Hubble rate approaches $H=0$ with a finite time in the case (a), while it diverges in the case (b).

This tells that our assumptions should be wrong and $\rho$ does not become infinite. If $\rho$ has an extremum, Eq. (107) tells that $H$ vanishes there since $\dot{\rho}=0$. In order to confirm whether the quantum effect moderates the singularity or not, we have numerically solved the background equations for the model where $\rho$ is exactly given by $f(\rho)=B \rho^{\beta}$ with $1 / 2<\beta<1$ and coefficients $b>0$, $b^{\prime}<0$ and $b^{\prime \prime}=0$. We find that the Hubble rate approaches zero with a finite time, as is similar to the case (a) in Fig. [5] Thus the presence of the quantum correction moderates the Big Rip singularity as well.

\section{Type II singularity}

Finally we consider the model $f(\rho)=C\left(\rho_{0}-\rho\right)^{-\gamma}$ with $\gamma>0$ as an example of the sudden future type singularity. By Eq. (62) the energy density $\rho$ in the classical case behaves as

$$
\rho \sim \rho_{0}-\left\{\kappa C \sqrt{3 \rho_{0}}(\gamma+1)\left(t_{s}-t\right)\right\}^{\frac{1}{\gamma+1}},
$$

around the singularity at $t=t_{s}$. Here we write $t_{0}$ in Eq. (62) as $t_{s}$. Using the Friedmann equation $H^{2}=$ $\kappa^{2} \rho / 3$, we find

$$
H \sim \kappa \sqrt{\frac{\rho_{0}}{3}}\left\{1-\frac{1}{2 \rho_{0}}\left[\kappa C \sqrt{3 \rho_{0}}(\gamma+1)\left(t_{s}-t\right)\right]^{\frac{1}{\gamma+1}}\right\} .
$$

Since $0<1 /(\gamma+1)<1, \dot{H}$ diverges at $t=t_{s}$ while $H$ is finite there.

Let us now include the quantum corrections. We assume the following form of $\rho$ around $t=t_{s}$ :

$$
\rho=\rho_{0}+\rho_{1}\left(t_{s}-t\right)^{\nu},
$$

with a positive constant $\nu$. Using Eq. (107), one gets

$$
H \sim \frac{\nu \rho_{1}^{1+\gamma}}{3(-C)}\left(t_{s}-t\right)^{-1+\nu(1+\gamma)}
$$

Since $\nu(1+\gamma)>0$, we can use the same approximate equation as in Eq. (110). This gives

$$
\nu=\frac{4}{2 \gamma+1}
$$

and

$$
\begin{aligned}
& \rho=\rho_{0}+\rho_{1}\left(t_{s}-t\right)^{\frac{4}{2 \gamma+1}}, \\
& H \propto\left(t_{s}-t\right)^{\frac{2 \gamma+3}{2 \gamma+1}} .
\end{aligned}
$$

Since $(2 \gamma+3) /(2 \gamma+1)$ is larger than 1 , not only $H$ but $\dot{H}$ are finite in the presence of quantum corrections. This is numerically confirmed in Fig. 6. Thus it is clear that quantum effects work to make the universe less singular or completely non-singular (basically, asymptotically deSitter). It was shown in Ref. 9] that this property also holds for scalar-field dark energy models.

Finally we should mention one point. When the quantum correction becomes important, this typically works to provide a negative energy density $\rho_{A}$ which nearly cancels with the energy density $\rho$ of dark energy. This is the reason why the Hubble rate does not diverge if we account for the quantum effect.

\section{DISCUSSION}

In summary, we discussed the fate of the (phantom) dark energy universe by assuming the equation of state (EOS) with a form: $p=-\rho-f(\rho)$. Our main interest is to clarify the structures of future singularities which appear with finite time $t_{s}$. We classified the types of singularities into four classes. The type I is the Big Rip singularity [5] at which all of $a, \rho$ and $p$ exhibit divergences. The type II corresponds to the sudden future singularity in [7] at which $a$ and $\rho$ are finite but $p$ diverges. The type III is similar to the Big Rip, but the scale factor is finite. The type IV is a mild singularity at which $a, \rho$, and $p$ are finite but higher derivatives of the Hubble rate diverge. The model given in Eq. (32) includes all of these singularities, which are investigated in details in Sec. III. In Sec. IV we adopted simpler forms of $f(\rho)$ which are obtained by considering limiting cases of the function (32) and studied the general structure of singularities. This is very helpful to understand when these finite-time singularities appear. 


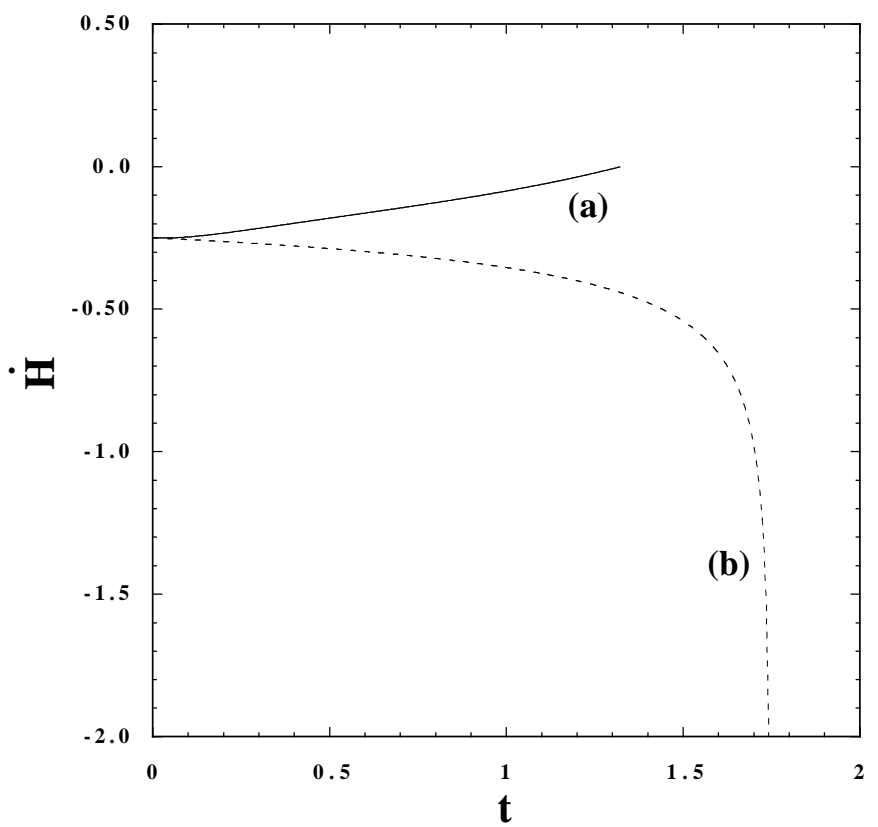

FIG. 6: The evolution of $\dot{H}$ for the model $f(\rho)=C\left(\rho-\rho_{0}\right)^{-\gamma}$ with $\gamma=1 / 2$ and $C<0$. We implement quantum effects in the case (a) with coefficients $b=0.5, b^{\prime}=-0.1$ and $b^{\prime \prime}=0$, whereas the case (b) does not account for such effects. We find that $\dot{H}$ approaches zero with a finite time in the case (a), while it diverges in the case (b).

In Sec. II we wrote the background equations in an autonomous form and showed that there exists one stable critical point if the constant $\operatorname{EOS}(w=p / \rho)$ of dark energy is less than -1 . It was also found that there is no stable node for $w>-1$. In Sec. $V$ we have numerically confirmed this property for the function (30) that asymptotically gives constant values of $w$. The singularity for $w<-1$ with the function (30) corresponds to the type I, which implies that the Big Rip singularity is a latetime stable attractor. We also performed phase-space analysis for the model (32) and found that the solutions approach "instantaneous" critical points introduced in Sec. II. We have numerically checked that the late-time solutions agree well with analytic estimations.

In Sec. VI a (phantom) dark energy scenario coupled to dark matter is investigated. We constructed the form of the coupling $Q$ that reproduces the background evolution (26) giving the Big Rip singularity at $t=t_{s}$, see Eqs. (66) and (73). Unlike the case of a single fluid, this allows a possibility to lead to the transition from $w>-1$ to $w<-1$ without using a double-valued function for $f(\rho)$. We also explicitly presented a two-scalar field model which gives the same singular behavior for the background evolution.

Finally, we accounted for the quantum correction coming from conformal anomaly and studied its effect to type I, II and III singularities. We found that this typically works to moderate the singularities or prevent them by providing a negative energy density. In this case the divergent behavior of the Hubble rate is prevented by the quantum effect, which is followed by the decrease of $H$ toward 0 . Therefore the presence of the quantum effect can drastically change the evolution of the universe. For instance, the new inflationary era in far future may be possible according to the conjecture of Refs. [6, [9].

It is quite clear that with the growth of the energy density near to singularity the effects of quantum gravity (string/M-theory) may become dominant. Hence, it will be extremely important to estimate the effect of dilatonic or modulus higher-order corrections 27] in dark energy models motivated by string theory, which we leave to future work. From another point, even if stringy effects stop the future singularity and possible phantom phase is transient, the growth of dark energy density may give its imprints to current universe. It would be a challenge to search for such imprints.

\section{ACKNOWLEDGEMENTS}

We thank J. Barrow for useful discussions. This research has been supported in part by the Ministry of Education, Science, Sports and Culture of Japan under the grant n.13135208 (S.N.), RFBR grant 03-01-00105, LRSS grant 1252.2003.2 and BFM2003-00620 grant (S.D.O).
[1] S. Hannestad and E. Mortsell, Phys. Rev. D 66, 063508 (2002) [arXiv:astro-ph/0205096];

A. Melchiorri, L. Mersini, C. J. Odman and M. Trodden, Phys. Rev. D 68, 043509 (2003) [arXiv:astro$\mathrm{ph} / 0211522]$;

J. Weller and A. M. Lewis, Mon. Not. Roy. Astron. Soc. 346, 987 (2003) [arXiv:astro-ph/0307104];

B. Feng, X. L. Wang and X. M. Zhang, Phys. Lett. B 607, 35 (2005) [arXiv:astro-ph/0404224];

P. S. Corasaniti, M. Kunz, D. Parkinson, E. J. Copeland and B. A. Bassett, Phys. Rev. D 70, 083006 (2004) [arXiv:astro-ph/0406608].

[2] R. R. Caldwell, Phys. Lett. B 545, 23 (2002)
[arXiv:astro-ph/9908168];

A. E. Schulz and M. J. White, Phys. Rev. D 64, 043514 (2001) [arXiv:astro-ph/0104112];

T. Chiba, T. Okabe and M. Yamaguchi, Phys. Rev. D 62, 023511 (2000) [arXiv:astro-ph/9912463];

B. Boisseau, G. Esposito-Farese, D. Polarski, A. A. Starobinsky, Phys. Rev. Lett. 85, 2236 (2000) [arXiv:gr-qc/0001066];

V. Faraoni, Int. J. Mod. Phys. D 64, 043514 (2002) [arXiv:gr-qc/0404078]; Phys. Rev. D 70, 044037 (2004) [arXiv:gr-qc/0407021];

G. W. Gibbons, hep-th/0302199;

S. Nojiri and S. D. Odintsov, Phys. Lett. B 562, 147 
(2003) [arXiv:hep-th/0303117]; Phys. Lett. B 571, 1 (2003) [arXiv:hep-th/0306212]; Phys. Lett. B 565, 1 (2003) [arXiv:hep-th/0304131];

P. Singh, M. Sami and N. Dadhich, Phys. Rev. D 68 023522 (2003) [arXiv:hep-th/0305110];

J.-g. Hao and X.-z. Li, Phys. Rev. D 69, 107303 (2004) [arXiv:hep-th/0303093]; Phys. Rev. D 70, 043529 (2004) [arXiv:astro-ph/0309746]; Phys. Lett. B 606, 7 (2005) [arXiv:astro-ph/0404154];

M. P. Dabrowski, T. Stachowiak and M. Szydlowski, Phys. Rev. D 68103519 (2003) [arXiv:hep-th/0307128]; L. P. Chimento and R. Lazkoz, Phys. Rev. Lett. 91, 211301 (2003) [arXiv:gr-qc/0307111]; arXiv:astro$\mathrm{ph} / 0405518$;

V. K. Onemli and R. P. Woodard, Class. Quant. Grav. 19, 4607 (2002) [arXiv:gr-qc/0204065]; Phys. Rev. D 70, 107301 (2004) [arXiv:gr-qc/0406098];

A. Feinstein and S. Jhingan, Mod. Phys. Lett. A 19, 457 (2004) [arXiv:hep-th/0304069].

[3] P. F. González-Díaz, arXiv:hep-th/0408225, to appear in TSPU Vestnik, 2004

E. Elizalde and J. Q. Hurtado, Mod. Phys. Lett. A 19, 29 (2004) [arXiv:gr-qc/0310128];

Y. Piao and E. Zhou, Phys. Rev. D 68083515 (2003) [arXiv:hep-th/0308080];

H. Stefancic, Phys. Lett. B 586, 5 (2004) [arXiv:astroph/0310904]; Eur. Phys. J. C 36, 523 (2004) [arXiv:astroph/0312484];

X. Meng and P. Wang, arXiv:hep-ph/0311070;

H. Q. Lu, arXiv:hep-th/0312082;

V. B. Johri, arXiv:astro-ph/0409161;

I. Brevik, S. Nojiri,S. D. Odintsov and L. Vanzo, Phys. Rev. D 70, 043520 (2004) [arXiv:hep-th/0401073];

J. Lima and J. S. Alcaniz, Phys. Lett. B 600191 (2004) [arXiv:astro-ph/0402265];

Z. Guo, Y. Piao and Y. Zhang, Phys. Lett. B 594247 (2004) [arXiv:astro-ph/0404225];

M. Bouhmadi-Lopez and J. Jimenez Madrid, arXiv:astro$\mathrm{ph} / 0404540$;

J. Aguirregabiria, L. P. Chimento and R. Lazkoz, Phys. Rev. D 70, 023509 (2004) [arXiv:astro-ph/0403157];

E. Babichev, V. Dokuchaev and Yu. Eroshenko, Class. Quant. Grav. 22, 143 (2005) [arXiv:astro-ph/0407190];

Y. Wei and Y. Tian, Class. Quant. Grav. 21, 5347 (2004) [arXiv:gr-qc/0405038];

A. Vikman, Phys. Rev. D 71, 023515 (2005) [arXiv:astroph/0407107];

B. Feng, M. Li, Y-S. Piao and X. m. Zhang, arXiv:astro$\mathrm{ph} / 0407432$;

S. M. Carroll, A. De Felice and M. Trodden, Phys. Rev. D 71, 023525 (2005) [arXiv:astro-ph/0408081];

C. Csaki, N. Kaloper and J. Terning, arXiv:astroph/0409596;

Y. Piao and Y. Zhang, Phys. Rev. D 70, 063513 (2004)

[arXiv:astro-ph/0401231],

S. Tsujikawa and M. Sami, Phys. Lett. B 603, 113 (2004)

[arXiv:hep-th/0409212];

H. Kim, arXiv:astro-ph/0408577;

X. m. Zhang, arXiv:hep-ph/0410292;

S.K. Srivastava, JHEP 0411, 070 (2004) [hepth/0411088];

P. Avelino, arXiv:astro-ph/0411033;

J. Q. Xia, B. Feng and X. M. Zhang, arXiv:astroph/0411501;
I. Ya. Aref'eva, A. S. Koshelev and S. Yu. Vernov, arXiv:astro-ph/0412619;

M. Bento, O. Bertolami, N. Santos and A. Sen, Phys. Rev. D 71, 063501 (2005) [arXiv:astro-ph/0412638];

S. Nojiri and S. D. Odintsov, Proc. Sci. WC 2004, 024 (2004) [archive:hep-th/0412030];

B. Gumjudpai, T. Naskar, M. Sami and S. Tsujikawa, arXiv:hep-th/0502191.

[4] E. Elizalde, S. Nojiri and S. D. Odintsov, Phys. Rev. D 70, 043539 (2004) [arXiv:hep-th/0405034].

[5] R. R. Caldwell, M. Kamionkowski and N. N. Weinberg, Phys. Rev. Lett. 91, 071301 (2003) [arXiv:astro$\mathrm{ph} / 0302506]$;

B. McInnes, JHEP 0208, 029 (2002) [arXiv:hepth/0112066];

P. Gonzalez-Diaz, Phys. Lett. B586, 1 (2004) [arXiv:astro-ph/0312579];

M. Sami and A. Toporensky, Mod. Phys. Lett. A19, 1509 (2004) [arXiv:gr-qc/0312009];

P. Gonzales-Diaz and C. Siguenza, Nucl. Phys. B697, 363 (2004) [arXiv:astro-ph/0407421];

L. P. Chimento and R. Lazkoz, Mod. Phys. Lett. A19, 2479 (2004) [arXiv:gr-qc/0405020];

G. Calcagni, Phys. Rev. D 71, 023511 (2005) [arXiv:grqc/0410027];

P. Wu and H. Yu, arXiv:astro-ph/0407424;

S. Nesseris and L. Perivolaropoulos, Phys. Rev. D 70, 123529 (2004) [arXiv:astro-ph/0410309];

P. Scherrer, arXiv:astro-ph/0410508;

Z. Guo,Y. Piao, X. Zhang and Y. Zhang, Phys. Lett. B 608177 (2005) [arXiv:astro-ph/0410654];

M. C. B. Abdalla, S. Nojiri and S. D. Odintsov, Class. Quant. Grav. 22, L35 (2005) [arXiv:hep-th/0409177].

Y. Wei, arXiv:gr-qc/0410050;

M. Dabrowski and T. Stachowiak, arXiv:hep-th/0411199.

[6] S. Nojiri and S. D. Odintsov, Phys. Rev. D 70, 103522 (2004) [arXiv:hep-th/0408170].

[7] J. D. Barrow, Class. Quant. Grav. 21, L79 (2004) [arXiv:gr-qc/0403084].

[8] J. D. Barrow, Class. Quant. Grav. 21, 5619 (2004) [arXiv:gr-qc/0409062].

S. Cotsakis and I. Klaoudatou, arXiv:gr-qc/0409022;

V. Sahni and Yu. Shtanov, JCAP 0311, 014 (2003) [arXiv:astro-ph/0202346];

K. Lake, Class. Quant. Grav. 21, L129 (2004) [arXiv:grqc/0407107];

M. Dabrowski, arXiv:gr-qc/0410033;

L. Fernandez-Jambrina and R. Lazkoz, Phys. Rev. D 70, 121503 (2004) [arXiv:gr-qc/0410124];

J. D. Barrow and C. Tsagas, arXiv:gr-qc/0411045;

[9] S. Nojiri and S. D. Odintsov, Phys. Lett. B 595, 1 (2004) [arXiv:hep-th/0405078].

[10] S. K. Srivastava, arXiv:hep-th/0411221;

H. Calderon and W. Hiscock, Class. Quant. Grav. 22, L23 (2005) [arXiv:gr-qc/0411134].

[11] E. J. Copeland, A. R. Liddle and D. Wands, Phys. Rev. D 57, 4686 (1998) [arXiv:gr-qc/9711068].

[12] S. C. C. Ng, N. J. Nunes and F. Rosati, Phys. Rev. D 64, 083510 (2001) [arXiv:astro-ph/0107321].

[13] E. J. Copeland, M. R. Garousi, M. Sami and S. Tsujikawa, Phys. Rev. D 71, 043003 (2005) [arXiv:hepth/0411192];

M. R. Garousi, M. Sami and S. Tsujikawa, arXiv:hepth/0412002. 
[14] H. Stefancic, arXiv:astro-ph/0411630.

[15] J. D. Barrow, Phys. Lett. B 180 (1986) 335; Nucl. Phys. B 310 (1988) 743.

[16] L. Amendola, Phys. Rev. D 62, 043511 (2000) [arXiv:astro-ph/9908023].

[17] L. Amendola, Phys. Rev. D 60, 043501 (1999) [arXiv:astro-ph/9904120].

[18] Z.-K. Guo, R.-G. Cai, Y.-Z. Zhang, arXiv:astro$\mathrm{ph} / 0412624$.

[19] W. Zimdahl and D. Pavon, Phys. Lett. B 521, 133 (2001) [arXiv:astro-ph/0105479].

[20] Z. K. Guo and Y. Z. Zhang, Phys. Rev. D 71, 023501 (2005) [arXiv:astro-ph/0411524].

[21] R. G. Cai and A. Wang, JCAP 0503, 002 (2005) [arXiv:hep-th/0411025].

[22] S. M. Carroll, M. Hoffman and M. Trodden, Phys. Rev. D 68, 023509 (2003) [arXiv:astro-ph/0301273].

[23] J. M. Cline, S. y. Jeon and G. D. Moore, Phys. Rev. D 70, 043543 (2004) [arXiv:hep-ph/0311312].
[24] F. Piazza and S. Tsujikawa, JCAP 0407, 004 (2004) [arXiv:hep-th/0405054].

[25] D. N. Spergel et al. [WMAP Collaboration], Astrophys. J. Suppl. 148, 175 (2003) [arXiv:astro-ph/0302209].

[26] S. Nojiri and S. D. Odintsov, Int. J. Mod. Phys. A 16, (2001) 3273 [archive:hep-th/0011115]; Class. Quant. Grav. 18, (2001), 5227 [arXiv:hep-th/0103078].

[27] M. Gasperini, M. Maggiore and G. Veneziano, Nucl. Phys. B 494, 315 (1997) [arXiv:hep-th/9611039];

R. Brustein and R. Madden, Phys. Rev. D 57, 712 (1998) [arXiv:hep-th/9708046];

C. Cartier, E. J. Copeland and R. Madden, JHEP 0001, 035 (2000) [arXiv:hep-th/9910169];

S. Tsujikawa, Phys. Lett. B 526, 179 (2002) [arXiv:grqc/0110124];

S. Tsujikawa, R. Brandenberger and F. Finelli, Phys. Rev. D 66, 083513 (2002) [arXiv:hep-th/0207228]. 